\title{
Automated, Highly-Accurate, Bug Assignment Using Machine Learning and Tossing Graphs
}

\author{
Pamela Bhattacharya $^{\mathrm{a}, *}$, Iulian Neamtiu ${ }^{\mathrm{a}}$, Christian R. Shelton ${ }^{\mathrm{a}}$ \\ ${ }^{a}$ Department of Computer Science and Engineering, University of California, Riverside, \\ $C A$, 92521, USA.
}

\begin{abstract}
Empirical studies indicate that automating the bug assignment process has the potential to significantly reduce software evolution effort and costs. Prior work has used machine learning techniques to automate bug assignment but has employed a narrow band of tools which can be ineffective in large, longlived software projects. To redress this situation, in this paper we employ a comprehensive set of machine learning tools and a probabilistic graph-based model (bug tossing graphs) that lead to highly-accurate predictions, and lay the foundation for the next generation of machine learning-based bug assignment. Our work is the first to examine the impact of multiple machine learning dimensions (classifiers, attributes, and training history) along with bug tossing graphs on prediction accuracy in bug assignment. We validate our approach on Mozilla and Eclipse, covering 856,259 bug reports and 21 cumulative years of development. We demonstrate that our techniques can achieve up to $86.09 \%$ prediction accuracy in bug assignment and significantly reduce tossing path lengths. We show that for our data sets the Naïve Bayes classifier coupled with product-component features, tossing graphs and incremental learning performs best. Next, we perform an ablative analysis by unilaterally varying classifiers, features, and learning model to show their relative importance of on bug assignment accuracy. Finally, we propose optimization techniques that achieve high prediction accuracy while reducing training and prediction time.
\end{abstract}

Keywords: Bug assignment, bug tossing, machine learning, empirical studies

\section{Introduction}

Software evolution has high associated costs and effort. A survey by the National Institute of Standards and Technology estimated that the annual cost of software bugs is about $\$ 59.5$ billion [1]. Some software maintenance studies

\footnotetext{
* Corresponding author

Email addresses: pamelab@cs.ucr.edu (Pamela Bhattacharya), neamtiu@cs.ucr.edu (Iulian Neamtiu), cshelton@cs.ucr.edu (Christian R. Shelton)
} 
indicate that maintenance costs are at least $50 \%$, and sometimes more than $90 \%$, of the total costs associated with a software product $[2,3]$, while other estimates place maintenance costs at several times the cost of the initial software version [4]. These surveys suggest that making the bug fixing process more efficient would reduce evolution effort and lower software production costs.

Most software projects use bug trackers to organize the bug fixing process and facilitate application maintenance. For instance, Bugzilla is a popular bug tracker used by many large projects, such as Mozilla, Eclipse, KDE, and Gnome [5]. These applications receive hundreds of bug reports a day; ideally, each bug gets assigned to a developer who can fix it in the least amount of time. This process of assigning bugs, known as bug assignment ${ }^{1}$, is complicated by several factors: if done manually, assignment is labor-intensive, time-consuming and fault-prone; moreover, for open source projects, it is difficult to keep track of active developers and their expertise. Identifying the right developer for fixing a new bug is further aggravated by growth, e.g., as projects add more components, modules, developers and testers [6], the number of bug reports submitted daily increases, and manually recommending developers based on their expertise becomes difficult. An empirical study by Jeong et al. [7] reports that, on average, the Eclipse project takes about 40 days to assign a bug to the first developer, and then it takes an additional 100 days or more to reassign the bug to the second developer. Similarly, in the Mozilla project, on average, it takes 180 days for the first assignment and then an additional 250 days if the first assigned developer is unable to fix it. These numbers indicate that the lack of effective, automatic assignment and toss reduction techniques results in considerably high effort associated with bug resolution.

Effective and automatic bug assignment can be divided into two sub-goals: (1) assigning a bug for the first time to a developer, and (2) reassigning it to another promising developer if the first assignee is unable to resolve it, then repeating this reassignment process (bug tossing) until the bug is fixed. Our findings indicate that at least $93 \%$ of all "fixed" bugs in both Mozilla and Eclipse have been tossed at least once (tossing path length $\geq 1$ ). Ideally, for any bug triage event, the bug should be resolved in a minimum number of tosses.

In this paper, we explore the use of machine learning toward effective and automatic bug assignment along three dimensions: the choice of classification algorithms, the software process attributes that are instrumental to constructing accurate prediction models, and the efficiency-precision trade-off. Our thorough exploration along these dimensions have lead us to develop techniques that achieve high levels of bug assignment accuracy and bug tossing reduction.

Similar to prior work, we test our approach on the fixed bug data sets for Mozilla and Eclipse. Our techniques achieve a bug assignment prediction ac-

\footnotetext{
${ }^{1}$ In the software maintenance literature, "bug triaging" is used as a broader term referring to bug assignment, bug validation, marking duplicate bugs, etc. In this paper, by bug triaging we mean bug assignment only, i.e., given a bug report that has been validated as a real bug, find the right developer whom the bug can be assigned to for resolution.
} 


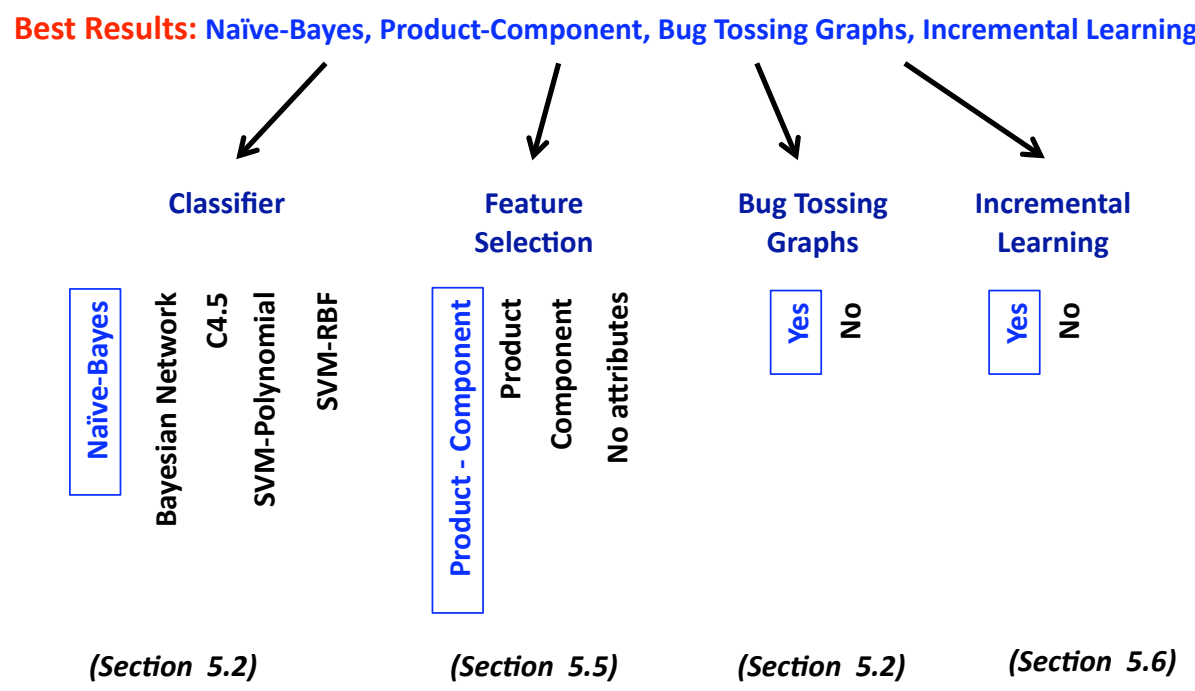

Figure 1: Overview our approach to ablative analysis for automating bug assignment: best case (boxed vertical text), attributes varied (unboxed vertical text), and the corresponding sections where the attributes and their importance are discussed.

curacy of up to $85 \%$ for Mozilla and $86 \%$ for Eclipse. We also find that using our approach reduces the length of tossing paths by up to $86 \%$ for correct predictions and improves the prediction accuracy by up to 10.78 percentage points compared to previous approaches. We demonstrate that on average, the highest prediction accuracy is achieved using a Naïve Bayes classifier, with products/components as attributes, with bug triaging graphs, and with incremental learning (aka intra-fold updates) as shown in Figure 1. We then follow a standard machine learning ablative analysis: ${ }^{2}$ we take our best case (top of the figure) and unilaterally vary the underlying attributes to show their relative importance in Section 5-the corresponding subsections are shown on the bottom of the figure. The primary goal of our work is to find the optimal set of machine learning techniques (classifiers, features, tossing graphs and incremental learning) to improve bug assignment accuracy in large projects and we show this optimal set for our data sets, Mozilla and Eclipse. The optimal set of techniques we report can change with changes in data sets for the same project or across other projects, or with changes in the underlying supervised learning algorithm and we address these issues as potential threats to validity of our approach in Section 6.

We now proceed to presenting the three main contributions of this work.

\footnotetext{
${ }^{2}$ Ablative analysis is a methodology to quantify the effects of each attribute in a multiattribute model.
} 
Wide range of classification algorithms. Machine learning is used for recommendation purposes in various areas such as climate prediction, stock market analysis, or prediction of gene interaction in bioinformatics [8]. Machine learning techniques, in particular classifiers, ${ }^{3}$ have also been employed earlier for automating bug assignment. These automatic bug assignment approaches $[9,10,11,12]$ use the history of bug reports and developers who fixed them to train a classifier. Later, when keywords from new bug reports are given as an input to the classifier, it recommends a set of developers who have fixed similar classes of bugs in the past and are hence considered potential bugfixers for the new bug. Prior work that has used machine learning techniques for prediction or recommendation purposes has found that prediction accuracy depends on the choice of classifier, i.e., a certain classifier outperforms other classifiers for a specific kind of a problem [8]. Previous studies [7, 9, 10, 11] only used a subset of text classifiers and did not aim at analyzing which is the best classifier for this problem. Our work is the first to examine the impact of multiple machine learning dimensions (classifiers, attributes, and training history) on prediction accuracy in bug assignment and tossing. In particular, this is the first study in the area of bug assignment to consider, and compare the performance of, a broad range of classifiers along with tossing graphs: Naïve Bayes Classifier, Bayesian Networks, C4.5 and Support Vector Machines.

Effective tossing graphs. Jeong et al. [7] have introduced tossing graphs for studying the process of tossing, i.e., bug reassignment; they proposed automating bug assignment by building bug tossing graphs from bug tossing histories. While classifiers and tossing graphs are effective in improving the prediction accuracy for assignment and reducing tossing path lengths, their accuracy is threatened by several issues: outdated training sets, inactive developers, and imprecise, single-attribute tossing graphs. Prior work [7] has trained a classifier with fixed bug histories; for each new bug report, the classifier recommends a set of potential developers, and for each potential developer, a tossing graphwhose edges contain tossing probabilities among developers - is used to predict possible re-assignees. However, the tossing probability alone is insufficient for recommending the most competent active developer (see Section 4.6.3 for an example). In particular, in open source projects it is difficult to keep track of active developers and their expertise. To address this, in addition to tossing probabilities, we label tossing graph edges with developer expertise and tossing graph nodes with developer activity, which help reduce tossing path lengths significantly. We demonstrate the importance of using these additional attributes in tossing graphs by performing a fine-grained per-attribute ablative analysis which reveals how much each attribute affects the prediction accuracy. We found that each attribute is instrumental for achieving high prediction accuracy, and overall they make pruning more efficient and improve prediction

\footnotetext{
${ }^{3} \mathrm{~A}$ classifier is a machine learning algorithm that can be trained using input attributes (also called feature vectors) and desired output classes; after training, when presented with a set of input attributes, the classifier predicts the most likely output class.
} 
accuracy by up to $22 \%$ points when compared to prediction accuracy obtained in the absence of the attributes.

Accurate yet efficient classification. Anvik's dissertation [13] has demonstrated that choosing a subset of training data can reduce the computation time during the classification process, while achieving similar prediction accuracies to using the entire data set; three methods - random, strict and tolerant, were employed for choosing a subset of training data set as we explain in Section 2. In our work, in addition to classification, we also use a probabilistic ranking function based on bug tossing graphs for developer recommendation. Since bug tossing graphs are time-sensitive, i.e., tossing probabilities change with time, the techniques used by Anvik are not applicable in our case (where bug reports were not sorted by time for selection). Therefore, in this paper, we propose to shorten the time-consuming classification process by selecting the most recent history for identifying developer expertise. As elaborated in Section 5.7 we found that by using just one third of all bug reports we could achieve prediction accuracies similar to the best results of our original experiments where we used the complete bug history. Therefore, our third contribution in this paper is showing how, by using a subset of bug reports, we can achieve accurate yet efficient bug classification that significantly reduces the computational effort associated with training.

Our paper is structured as follows. In Section 2 we discuss prior work and how it relates to our approach. In Section 3 we define terms and techniques used in bug assignment. In Section 4 we elaborate on our contributions, techniques and implementation details. We present our experimental setup and results in Section 5. Finally, we discuss threats to validity of our study in Section 6.

\section{Related Work}

\subsection{Machine Learning and Information Retrieval Techniques}

Cubranic et al. [10] were the first to propose the idea of using text classification methods (similar to methods used in machine learning) to semi-automate the process of bug assignment. They used keywords extracted from the title and description of the bug report, as well as developer ID's as attributes, and trained a Naïve Bayes classifier. When presented with new bug reports, the classifier suggests one or more potential developers for fixing the bug. Their method used bug reports for Eclipse from January 1, 2002 to September 1, 2002 for training, and reported a prediction accuracy of up to $30 \%$. While we use classification as a part of our approach, in addition, we employ incremental learning and tossing graphs to reach higher accuracy. Moreover, our data sets are much larger, covering the entire lifespan of both Mozilla (from May 1998 to March 2010) and Eclipse (from October 2001 to March 2010).

Anvik et al. [9] improved the machine learning approach proposed by Cubranic et al. by using filters when collecting training data: (1) filtering out bug reports labeled "invalid," "wontfix," or "worksforme," (2) removing developers who no 
longer work on the project or do not contribute significantly, and (3) filtering developers who fixed less than 9 bugs. They used three classifiers, SVM, Naïve Bayes and C4.5. They observed that SVM (Support Vector Machines) performs better than the other two classifiers and reported prediction accuracy of up to $64 \%$. Our ranking function (as described in Section 4) obviates the need to filter bugs. Similar to Anvik et al., we found that filtering bugs which are not "fixed" but "verified" or "resolved" leads to higher accuracy. They report that their initial investigation in incremental learning did not have a favorable outcome, whereas incremental learning helps in our approach; in Section 5 we explain the discrepancy between their findings and ours.

Anvik's dissertation [13] presented seminal work in building recommendation systems for automating the bug assignment process using machine learning algorithms. His work differentiated between two kinds of triage decisions: (1) repository-oriented decisions (determining whether a bug report is meaningful, such as if the report is a duplicate or is not reproducible), and (2) developmentoriented decisions (finding out whether the product/component of a bug report determines the developer the report is assigned to). They used a wide range of machine learning algorithms (supervised classification: Naïve Bayes, SVM, C4.5, Conjunctive Rules, and Nearest Neighbor and unsupervised classification: Expectation Maximization) for evaluating the proposed model and suggested how a subset of the bug reports chosen randomly or user-selected threshold could be used for classifier training. Similar to Anvik, we show how using four supervised classifiers (Naïve Bayes, Bayesian Networks, SVM, and C4.5) and a subset of training data can be used to improve bug assignment accuracy. In addition to classification, we also use a ranking function based on bug tossing graphs for developer recommendation and perform an ablative analysis to determine the significance of the attributes in the ranking function; Anvik's dissertation neither employ bug tossing graphs nor performs any ablative analysis. Anvik proposed three types of subset training data selection: random (100 bug reports where chosen in each iteration until desired prediction accuracy was achieved), strict (number of bug reports for each developer where determined depending on his lifetime contribution) and tolerant (number of bug reports were chosen randomly and was proportional to a developer's contribution); in contrast, we used a chronologically-backtracking method to find out the subset of bug reports that can be used to efficiently predict bug triagers instead of random selection. For evaluating their framework, they used bug reports from 5 projects: Firefox, Eclipse, gcc, Mylyn, Bugzilla. Their prediction accuracy is as follows: $75 \%$ for Firefox (by using 6,356 bug reports for training and 152 bug reports for validation) and $70 \%$ for Eclipse (by using 3,338 bug reports for training and $64 \mathrm{bug}$ reports for validation). Our work differs significantly from theirs in two ways: first, we use a different data set for our training and validation and we use all Mozilla products instead of Firefox alone, and second, we propose incremental machine learning based and probabilistic graph-based approach for bug assignment. By using all products in Mozilla and Eclipse, we can prune developer expertise further by our ranking function which leads to higher prediction accuracy. 
Canfora et al. used probabilistic text similarity [12] and indexing developers/modules changed due to bug fixes [14] to automate bug assignment. When using information retrieval based bug assignment, they report up to 50\% Top 1 recall accuracy and when indexing source file changes with developers they achieve 30\%-50\% Top 1 recall for KDE and 10\%-20\% Top 1 recall for Mozilla.

Podgurski et al. [15] also used machine learning techniques to classify bug reports but their study was not targeted at bug assignment; rather, their study focused on classifying and prioritizing various kinds of software faults.

Lin et al. [16] conducted machine learning-based bug assignment on a proprietary project, SoftPM. Their experiments were based on 2,576 bug reports. They report $77.64 \%$ average prediction accuracy when considering module ID (the module a bug belongs to) as an attribute for training the classifier; the accuracy drops to $63 \%$ when module ID is not used. Their finding is similar to our observation that using product-component information for classifier training improves prediction accuracy.

Lucca et al. [17] used information retrieval approaches to classify maintenance requests via classifiers. However, the end goal of their approach is bug classification, not bug assignment. They achieved up to $84 \%$ classification accuracy by using both split-sample and cross-sample validation techniques.

Matter et al. [18] model a developer's expertise using the vocabulary found in the developer's source code. They recommend potential developers by extracting information from new bug reports and looking it up in the vocabulary. Their approach was tested on 130,769 Eclipse bug reports and reported prediction accuracies of $33.6 \%$ for top 1 developers and $71 \%$ for top 10 developers.

\subsection{Incremental Learning}

Bettenburg et al. [11] demonstrate that duplicate bug reports are useful in increasing the prediction accuracy of classifiers by including them in the training set for the classifier along with the master reports of those duplicate bugs. They use folding to constantly increase the training data set during classification, and show how this incremental approach achieves prediction accuracies of up to $56 \%$; they do not need tossing graphs, because reducing tossing path lengths is not one of their goals. We use the same general approach for the classification part, though we improve it by using more attributes in the training data set; in addition, we evaluate the accuracy of multiple text classifiers; and we achieve higher prediction accuracies.

\subsection{Tossing Graphs}

Jeong et al. [7] introduced the idea of using bug tossing graphs to predict a set of suitable developers for fixing a bug. They used classifiers and tossing graphs (Markov-model based) to recommend potential developers. We use finegrained, intra-fold updates and extra attributes for classification; our tossing graphs are similar to theirs, but we use additional attributes on edges and nodes as explained in Section 4. The set of attributes we use help improve prediction accuracy and further reduce tossing lengths, as described in Sections 5.2 and 5.3. 
We also perform an ablative analysis to demonstrate the importance of using additional attributes in tossing graphs and tossee ranking.

\section{Preliminaries}

We first define several machine learning and bug assignment concepts that form the basis of our approach.

\subsection{Machine Learning for Bug Categorization}

Classification is a supervised machine learning technique for deriving a general trend from a training data set. The training data set (TDS) consists of pairs of input objects (called feature vectors), and their respective target outputs. The task of the supervised learner (or classifier) is to predict the output given a set of input objects, after being trained with the TDS. Feature vectors for which the desired outputs are already known form the validation data set (VDS) that can be used to test the accuracy of the classifier. A bug report contains a description of the bug and a list of developers that were associated with a specific bug, which makes text classification applicable to bug assignment. Machine learning techniques were used by previous bug assignment works $[9,10,11]$ : archived bug reports form feature vectors, and the developers who fixed the bugs are the outputs of the classifier. Therefore, when a new bug report is provided to the classifier, it predicts potential developers who can fix the bug based on their bug fixing history.

Feature vectors. The accuracy of a classifier is highly dependent on the feature vectors in the TDS. Bug titles and summaries have been used earlier to extract the keywords that form feature vectors. These keywords are extracted such that they represent a specific class of bugs. For example, if a bug report contains words like "icon," "image," or "display," it can be inferred that the bug is related to application layout, and is assigned to the "layout" class of bugs. We used multiple text classification techniques (tf-idf, stemming, stop-word and non-alphabetic word removal [19]) to extract relevant keywords from the actual bug report; these relevant keywords constitute a subset of the attributes used to train the classifier.

\subsubsection{Text Classification Algorithms}

We now briefly describe each classifier we used.

Naïve Bayes Classifier. Naïve Bayes is a probabilistic technique that uses Bayes' rule of conditional probability to determine the probability that an instance belongs to a certain class. Bayes' rule states that "the probability of a class conditioned on an observation is proportional to the prior probability of the class times the probability of the observation conditioned on the class" and can be denoted as follows: 


$$
P(\text { class } \mid \text { observation })=\frac{P(\text { observation } \mid \text { class }) * P(\text { class })}{P(\text { observation })}
$$

For example, if the word concurrency occurs more frequently in the reports resolved by developer $A$ than in the reports resolved by developer $B$, the classifier would predict $A$ as a potential fixer for a new bug report containing the word concurrency. "Naïve Bayes" is so called because it makes the strong assumption that features are independent of each other, given the label (the developer who resolved the bug). Even though this assumption does not always hold, Naïve Bayes-based recommendation or prediction performs well in practice [20].

Bayesian Networks. A Bayesian Network [21] is a probabilistic model that is used to represent a set of random variables and their conditional dependencies by using a directed acyclic graph (DAG). Each node in the DAG denotes a variable, and each edge corresponds to a potential direct dependence relationship between a pair of variables. Each node is associated with a conditional probability table $(\mathrm{CPT})$ which gives the probability that the corresponding variable takes on a particular value given the values of its parents.

C4.5. The C4.5 algorithm [22] builds a decision tree based on the attributes of the instances in the training set. A prediction is made by following the appropriate path through the decision tree based on the attribute values of the new instance. C4.5 builds the tree recursively in a greedy fashion. Each interior node of the tree is selected to maximize the information gain of the decision at that node as estimated by the training data. The information gain is a measure of the predictability of the target class (developer who will resolve the bug report) from the decisions made along the path from the root to this node in the tree. The sub-trees end in leaf nodes at which no further useful distinctions can be made and thus a particular class is chosen.

Support Vector Machines. An SVM (Support Vector Machine [23]) is a supervised classification algorithm that finds a decision surface that maximally separates the classes of interest. That is, the closest points to the surface on each side are as far as possible from the decision surface. It employs kernels to represent non-linear mappings of the original input vectors. This allows it to build highly non-linear decision surfaces without an explicit representation of the non-linear mappings. Four kinds of kernel functions are commonly used: Linear, Polynomial, Gaussian Radial Basis Function (RBF) and Sigmoid. In our study we use Polynomial and RBF functions as they have been found to be most effective in text classification.

\subsection{Folding}

Early bug assignment approaches $[7,9,10]$ divided the data set into two subsets: $80 \%$ for TDS and $20 \%$ for VDS. Bettenburg et al. [11] have used folding (similar to split-sample validation techniques from machine learning [8]) in the context of detecting duplicate bug reports. In a folding-based training and 


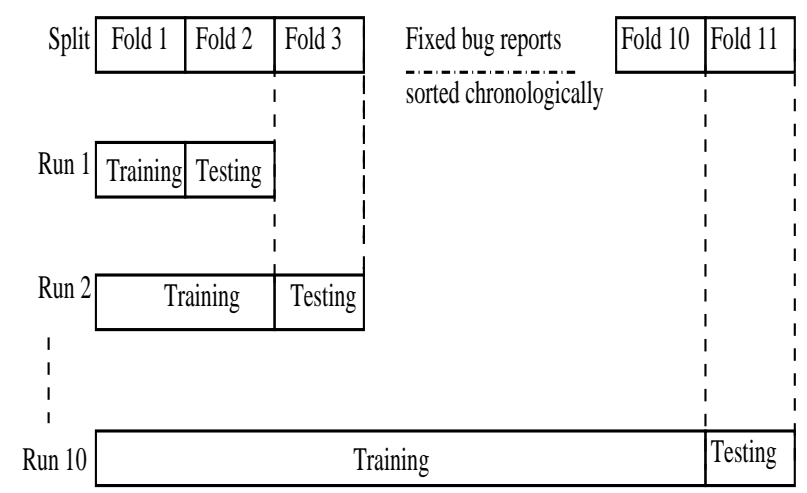

Figure 2: Folding techniques for classification as used by Bettenburg et al.

validation approach, also known as cross-validation, (illustrated in Figure 2), the algorithm first collects all bug reports to be used for TDS, ${ }^{4}$ sorts them in chronological order (based on the fixed date of the bug) and then divides them into $n$ folds. In the first run, fold 1 is used to train the classifier and then to predict the VDS. ${ }^{5}$ In the second run, fold 2 bug reports are added to TDS. In general, after validating the VDS from fold $n$, that VDS is added to the TDS for validating fold $n+1$. To reduce experimental bias [8], similar to Bettenburg et al., we chose $n=11$ and carried out 10 iterations of the validation process using incremental learning. Note that incremental learning is not a contribution of our work; incremental learning is a standard technique to improve the prediction accuracy in any supervised or unsupervised learning algorithms in machine learning [24]. Rather, we show that, similar to other software maintenance problems like duplicate bug detection [11], fine-grained incremental learning is important for improving bug assignment accuracy, i.e., to have the classifier trained with most recent data (or bug reports). Therefore, we only use folding to compare our work with prior studies in automatic bug assignment where split-sample validation was used; though our best result was achieved using fine-grained incremental learning.

\subsection{Goal-oriented Tossing Graphs}

When a bug is assigned to a developer for the first time and she is unable to fix it, the bug is assigned (tossed) to another developer. Thus a bug is tossed from one developer to another until a developer is eventually able to fix it. Based on these tossing paths, goal-oriented tossing graphs were proposed by Jeong et al .[7]; for the rest of the paper, by "tossing graph" we refer to a goal-oriented tossing graph. Tossing graphs are weighted directed graphs such that each node represents a developer, and each directed edge from $D_{1}$ to $D_{2}$

\footnotetext{
${ }^{4}$ Training Data Set (TDS) used to train the classifier; see Section 3.1 for more details.

${ }^{5}$ Validation Data Set (VDS) used to validate the classifier; see Section 3.1 for more details.
} 
represents the fact that a bug assigned to developer $D_{1}$ was tossed and eventually fixed by developer $D_{2}$. The weight of an edge between two developers is the probability of a toss between them, based on bug tossing history. We denote a tossing event from developer $D$ to $D_{j}$ as $D \hookrightarrow D_{j}$. The tossing probability (also known as the transaction probability) from developer $D$ to $D_{j}$ is defined by the following equation where $k$ is the total number of developers who fixed bugs that were tossed from $D$ :

$$
\operatorname{Pr}\left(D \hookrightarrow D_{j}\right)=\frac{\#\left(D \hookrightarrow D_{j}\right)}{\sum_{i=1}^{k} \#\left(D \hookrightarrow D_{i}\right)}
$$

\begin{tabular}{|c|c|c|c|c|c|c|c|}
\hline \multicolumn{8}{|c|}{ Tossing paths } \\
\hline \multicolumn{8}{|c|}{$\begin{array}{l}A \rightarrow B \rightarrow C \rightarrow D \\
A \rightarrow E \rightarrow D \rightarrow C \\
A \rightarrow B \rightarrow E \rightarrow D \\
C \rightarrow E \rightarrow A \rightarrow D \\
B \rightarrow E \rightarrow D \rightarrow F\end{array}$} \\
\hline \multirow{3}{*}{$\begin{array}{c}\text { Developer } \\
\text { who tossed } \\
\text { the bug }\end{array}$} & \multirow{3}{*}{$\begin{array}{c}\text { Total } \\
\text { tosses }\end{array}$} & \multicolumn{6}{|c|}{ Developers who fixed the bug } \\
\hline & & \multicolumn{2}{|c|}{$C$} & \multicolumn{2}{|c|}{$D$} & \multicolumn{2}{|c|}{$F$} \\
\hline & & $\#$ & $\operatorname{Pr}$ & $\#$ & $\operatorname{Pr}$ & $\#$ & $\operatorname{Pr}$ \\
\hline$A$ & 4 & 1 & 0.25 & 3 & 0.75 & 0 & 0 \\
\hline$B$ & 3 & 0 & 0 & 2 & 0.67 & 1 & 0.33 \\
\hline$C$ & 2 & - & - & 2 & 1.00 & 0 & 0 \\
\hline$D$ & 2 & 1 & 0.50 & - & - & 1 & 0.50 \\
\hline$E$ & 4 & 1 & 0.25 & 2 & 0.50 & 1 & 0.25 \\
\hline
\end{tabular}

Table 1: Tossing paths and probabilities as used by Jeong et al.

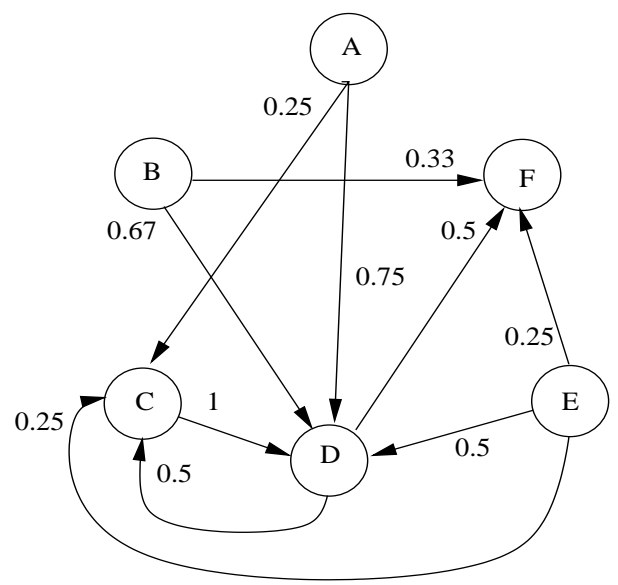

Figure 3: Tossing graph built using tossing paths in Table 1. 
In this equation, the numerator is the number $m$ of tosses from developer $D$ to $D_{j}$ such that $D_{j}$ fixed the bug, while the denominator is the total number of tosses from $D$ to any other developer $D_{i}$ such that $D_{i}$ fixed the bug. Note that if $k=0$ for any developer $D$, it denotes that $D$ has no outgoing edge in the bug tossing graph. To illustrate this, in Table 1 we provide sample tossing paths and show how toss probabilities are computed. For example, developer $A$ has tossed four bugs in all, three that were fixed by $D$ and one that was fixed by $C$, hence $\operatorname{Pr}(A \hookrightarrow D)=0.75, \operatorname{Pr}(A \hookrightarrow C)=0.25$, and $\operatorname{Pr}(A \hookrightarrow F)=0$. Note that developers who did not toss any bug (e.g., $F$ ) do not appear in the first column, and developers who did not fix any bugs (e.g., $A$ ) do not have a probability column. In Figure 3, we show the final tossing graph built using the computed tossing probabilities. It is common in open source projects that when a bug in a module is first reported, the developers associated with that module are included in the list of assignees by default. The purpose of our automatic bug assignment approach is, given a bug report, to predict developers who could be potential fixers and email them, so that human intervention is reduced as much as possible.

Prediction accuracy. If the first developer in our prediction list matches the actual developer who fixed the bug, we have a hit for the Top 1 developer count. Similarly, if the second developer in our prediction list matches the actual developer who fixed the bug, we have a hit for the Top 2 developer count. For example, if there are 100 bugs in the VDS and for 20 of those bugs the actual developer is the first developer in our prediction list, the prediction accuracy for Top 1 is $20 \%$; similarly, if the actual developer is in our Top 2 for 60 bugs, the Top 2 prediction accuracy is $60 \%$.

\section{Methodology}

\subsection{Choosing Effective Classifiers and Features}

In this section we discuss appropriate selection of machine learning algorithms and feature vectors for improving the classification process.

\subsubsection{Choosing the Right Classifier}

Various approaches that use machine learning techniques for prediction or recommendation purposes have found that prediction accuracy depends on the choice of classifier, i.e., for a specific kind of a problem, a certain classifier outperforms other classifiers [8]. Previous bug classification and assignment studies $[7,9,10,11]$ only used a subset of text classifiers and did not aim at analyzing which classifier works best for bug assignment. Our work is the first study to consider an extensive set of classifiers which are commonly used for text classification: Naïve Bayes Classifier, Bayesian Networks, C4.5 and two types of SVM classifiers (Polynomial and RBF). We found that for bug assignment it is not possible to select one classifier which is better than the rest, either for a specific project or for any project in general. Since classifier 
performance is also heavily dependent on the quality of bug reports, in general we could not propose choosing a specific classifier a priori for a given project. Interestingly, computationally-intensive classification algorithms such as C4.5 and SVM do not consistently outperform simpler algorithms such as Naïve Bayes and Bayesian Networks. We provide details of our prediction accuracy using each classifier in Section 5.2.

\subsubsection{Feature Selection}

Classifier performance is heavily dependent on feature selection [8]. Prior work $[9,10,11]$ has used keywords from the bug report and developer name or ID as features (attributes) for the training data sets; we also include the product and component the bug belongs to. For extracting relevant words from bug reports, we employ tf-idf, stemming, stop-word and non-alphabetic word removal [19]. We use the Weka toolkit [25] to remove stop words and form the word vectors for the dictionary (via the StringtoWordVector class with tf-idf enabled).

\subsection{Incremental Learning}

Prior work $[7,11]$ has used inter-fold updates, i.e., the classifier and tossing graphs are updated after each fold validation, as shown in Figure 4(a). With inter-fold updates, after validating the VDS from fold $n$, the VDS is added to the TDS for validating fold $n+1$. However, consider the example when the TDS contains bugs $1-100$ and the VDS contains bugs 101-200. When validating bug 101, the classifier and tossing graph are trained based on bugs 1-100, but from bug 102 onwards, the classifier and tossing graph are not up-to-date any more because they do not incorporate the information from bug 101. As a result, when the validation sets contain thousands of bugs, this incompleteness affects prediction accuracy. Therefore, to achieve high accuracy, it is essential that the classifier and tossing graphs be updated with the latest bug fix; we use a fine-grained, intra-fold updating technique (i.e., incremental learning) for this purpose.

We now proceed to describing intra-fold updating. After the first bug in the validation fold has been used for prediction and accuracy has been measured, we add it to the TDS and re-train the classifier as shown in Figure 11(b). We also update the tossing graphs by adding the tossing path of the just-validated bug. This guarantees that for each bug in the validation fold, the classifier and the tossing graphs incorporate information about all preceding bugs.

\subsection{Multi-featured Tossing Graphs}

Tossing graphs are built using tossing probabilities derived by analyzing bug tossing histories, as explained in Section 3.3. Jeong et al. [7] determined potential tossees as follows: if developer $A$ has tossed more bugs to developer $B$ than to developer $D$, in the future, when $A$ cannot resolve a bug, the bug will be tossed to $B$, i.e., tossing probabilities determine tossees. However, this approach might be inaccurate in certain situations: suppose a new bug belonging to class 

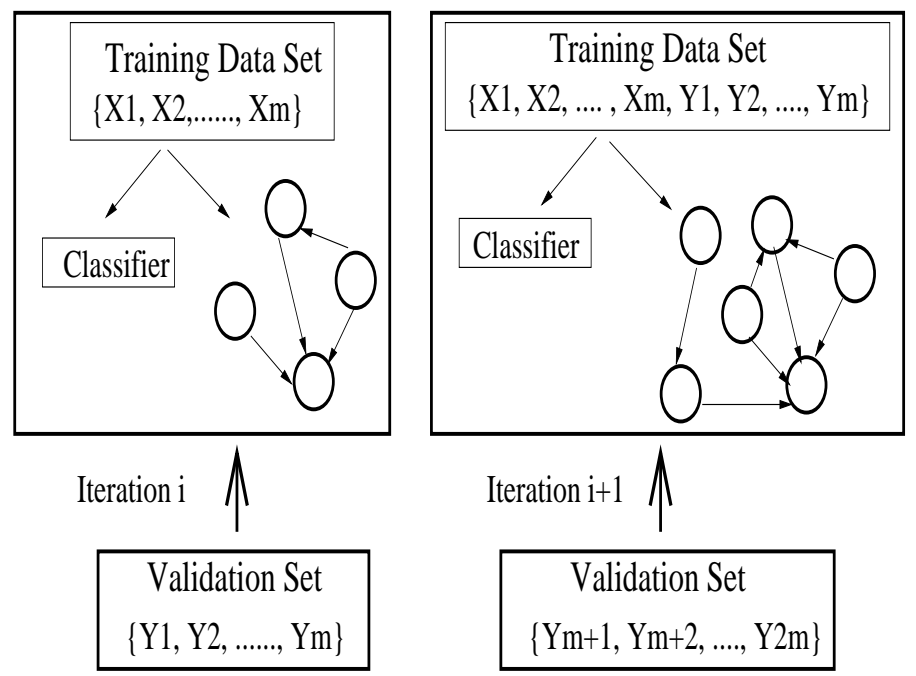

(a) Updates after each validation set (Bettenburg et al.)

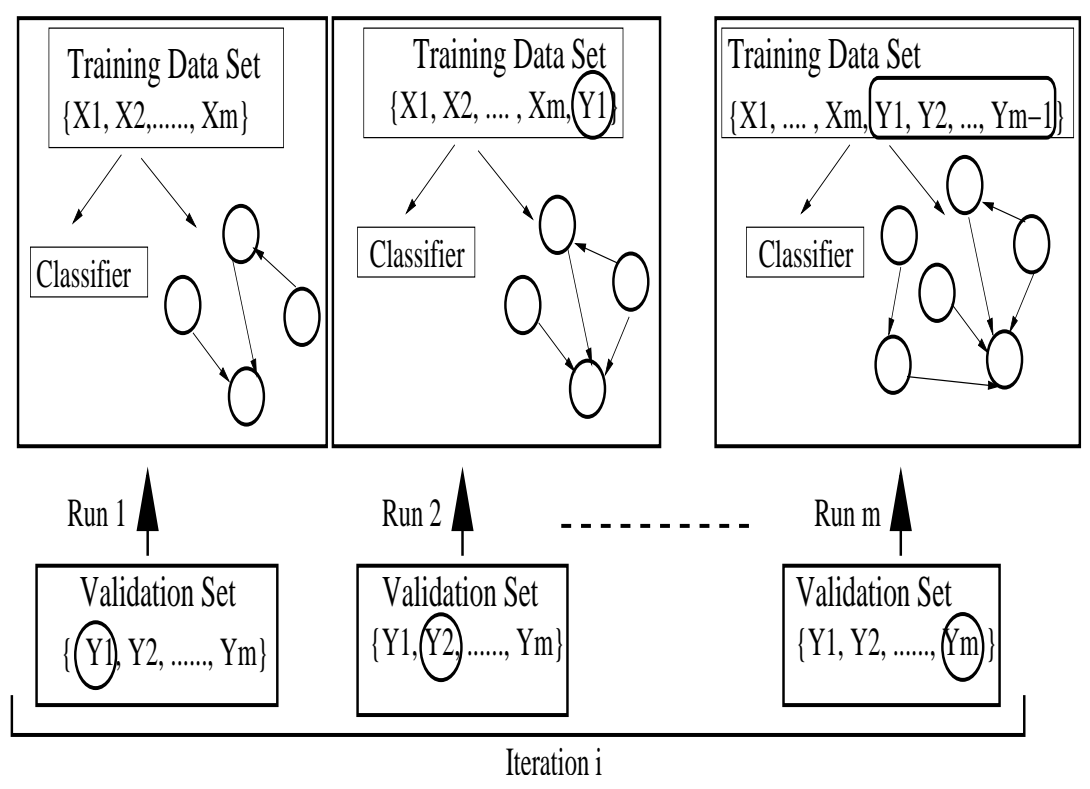

(b) Updates after each bug (our approach)

Figure 4: Comparison of training and validation techniques.

$K_{1}$ is reported, and developer $A$ was assigned to fix it, but he is unable to fix it; developer $B$ has never fixed any bug of type $K_{1}$, while $D$ has fixed 10 bugs of type $K_{1}$. The prior approach would recommend $B$ as the tossee, although $D$ is more likely to resolve the bug than $B$. Thus, although tossing graphs reveal 
tossing probabilities among developers, they should also contain information about which classes of bugs were passed from one developer to another; we use multi-feature tossing graphs to capture this information.

\begin{tabular}{|c|c|c|c|c|c|c|c|}
\hline Product & Component & \multicolumn{6}{|c|}{ Tossing paths } \\
\hline $\begin{array}{l}P_{1} \\
P_{1} \\
P_{2} \\
P_{1} \\
P_{1} \\
P_{1}\end{array}$ & $\begin{array}{l}C_{1} \\
C_{3} \\
C_{5} \\
C_{3} \\
C_{1} \\
C_{3}\end{array}$ & & & $\begin{array}{l}A- \\
\rightarrow \\
\rightarrow \\
\rightarrow \\
\rightarrow \\
\rightarrow\end{array}$ & $\begin{array}{l}B \rightarrow \\
\rightarrow B \\
\rightarrow D \\
\rightarrow A \\
\rightarrow E \\
\rightarrow F\end{array}$ & $\begin{array}{l}\vec{C} \\
\rightarrow E \\
\rightarrow C \\
\rightarrow I \\
\rightarrow C \\
\rightarrow I\end{array}$ & \\
\hline \multirow{3}{*}{$\begin{array}{c}\text { Developer } \\
\text { bug } \\
\text { assigned }\end{array}$} & \multirow{3}{*}{$\begin{array}{l}\text { Total } \\
\text { tosses }\end{array}$} & \multicolumn{6}{|c|}{ Developers who fixed the bug } \\
\hline & & \multicolumn{2}{|c|}{$C$} & \multicolumn{2}{|c|}{$D$} & \multicolumn{2}{|c|}{$E$} \\
\hline & & $\#$ & $P r$ & $\#$ & $\operatorname{Pr}$ & $\#$ & $P r$ \\
\hline$A$ & 6 & 3 & 0.5 & 2 & 0.33 & 1 & 0.17 \\
\hline \multicolumn{4}{|c|}{ Developer } & \multicolumn{4}{|c|}{$\begin{array}{c}\text { Last Activity } \\
\text { (in days) }\end{array}$} \\
\hline & $\begin{array}{l}A \\
C \\
D \\
E\end{array}$ & & & & & $\begin{array}{l}20 \\
70 \\
50 \\
150\end{array}$ & \\
\hline
\end{tabular}

Table 2: Example of tossing paths, associated tossing probabilities and developer activity.

Another problem with the classifier- and tossing graph-based approaches is that it is difficult to identify retired or inactive developers. This issue is aggravated in open source projects: when developers work voluntarily, it is difficult to keep track of the current set of active developers associated with the project. Anvik et al. [9] and Jeong et al. [7] have pointed out this problem and proposed solutions. Anvik et al. use a heuristic to filter out developers who have contributed fewer than 9 bug resolutions in the last 3 months of the project. Jeong et al. assume that, when within a short time span many bugs get tossed from a developer $D$ to others, leading to an increase in the number of outgoing edges in the tossing graph from $D$ 's node, $D$ is a potentially retired developer. They suggest that this information can be used in real-world scenarios by managers to identify potentially inactive developers. Therefore, in their automatic bug assignment approach they still permit assignment of bugs to inactive developers, which increases the length of the predicted tossing paths. In contrast, we restrict potential assignees to active developers only, and do so with a minimum number of tosses.

The tossing graphs we build have additional labels compared to Jeong et al.: for each bug that contributes to an edge between two developers, we attach the bug class (product and component) ${ }^{6}$ to that edge; moreover, for each developer

\footnotetext{
${ }^{6}$ Products are smaller projects within a large project. Components are sub-modules in
} 
in the tossing graph, we maintain an activity count, i.e., the difference between the date of the bug being validated and the date of the last activity of that developer.

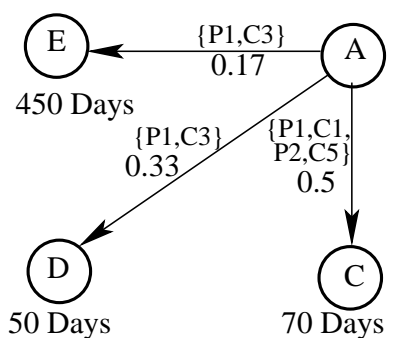

Figure 5: Multi-feature tossing graph (partial) derived from data in Table 2.

\subsubsection{Building Multi-feature Tossing Graphs}

As discussed earlier in Section 4.3, tossing probabilities are a good start toward indicating potential bug fixers, but they might not be appropriate at all times. Therefore, the tossing graphs we generate have three labels in addition to the tossing probability: bug product and bug component on each edge, and number of days since a developer's last activity on each node. For example, consider three bugs that have been tossed from $D_{1}$ to $D_{2}$ and belong to three different product-component sets: $\left\{P_{1}, C_{1}\right\},\left\{P_{1}, C_{3}\right\}$, and $\left\{P_{2}, C_{5}\right\}$. Therefore, in our tossing graph, the product-component set for the edge between $D_{1}$ and $D_{2}$ is $\left\{\left\{P_{1}, C_{1}\right\},\left\{P_{1}, C_{3}\right\},\left\{P_{2}, C_{5}\right\}\right\}$. Maintaining these additional attributes is also helpful when bugs are re-opened. Both developer expertise and tossing histories change over time, hence it is important to identify the last fixer for a bug and a potential tossee after the bug has been re-opened.

We now present three examples that demonstrate our approach and show the importance of multi-feature tossing graphs. The examples are based on the tossing paths, the product-component the bug belongs to, and the developer activity, as shown in Table 2. Suppose that at some point in our recommendation process for a specific bug, the classifier returns $A$ as the best developer for fixing the bug. However, if $A$ is unable to resolve it, we need to use the tossing graph to find the next developer. We will present three examples to illustrate which neighbor of $A$ to choose, and how the selection depends on factors like bug source and developer activity, in addition to tossing probability. For the purpose of these examples, we just show a part of the tossing graph built from the tossing paths shown in Table 2; we show the node for developer $A$ and its neighbors in the tossing graph in Figure 5, as the tossee selection is dependent on these nodes alone.

a product. For example, Firefox is a product in Mozilla and Bookmarks is a component of Firefox. 
Example I. Suppose we encounter a new bug $B_{1}$ belonging to product $P_{1}$ and component $C_{5}$, and the classifier returns $\mathrm{A}$ as the best developer for fixing the bug. If $\mathrm{A}$ is unable to fix it, by considering the tossing probability and product-component match, we conclude that it should be tossed to C.

Example II. Consider a bug $B_{2}$ belonging to product $P_{1}$ and component $C_{3}$. If $A$ is unable to fix it, although $C$ has a higher transaction probability than $D$, because $D$ has fixed bugs earlier from product $P_{1}$ and component $C_{3}$, he is more likely to fix it than $C$. Hence in this case the bug gets tossed from $A$ to $D$.

Example III. Based on the last active count for $E$ in Figure 5, i.e., 450 days, it is likely that $E$ is a retired developer. In our approach, if a developer has been inactive for more than 100 days, ${ }^{7}$ we choose the next potential neighbor (tossee) from the reference node $A$. For example, consider bug $B_{3}$ which belongs to product $P_{1}$ and component $C_{3}$, which has been assigned to $A$ and we need to find a potential tossee when $A$ is unable to resolve it. We should never choose $E$ as a tossee as he is a potential retired developer and hence, in this particular case, we choose $C$ as the next tossee. We also use activity counts to prune inactive developers from classifier recommendations. For example, if the classifier returns $n$ recommendations and we find that the $i^{\text {th }}$ developer is probably retired, we do not select him, and move on to the $(i+1)^{s t}$ developer.

\subsubsection{Ranking Function}

As explained with examples in Section 4.3.1, the selection of a tossee depends on multiple factors. We thus use a ranking function to rank the tossees and recommend a potential bug-fixer. We first show an example of our developer prediction technique for a real bug from Mozilla and then present the ranking function we use for prediction.

Example (Mozilla bug 254967). For this particular bug, the first five developers predicted by the Naïve Bayes classifier are \{bugzilla, fredbezies, myk, tanstaafl, ben.bucksch\}. However, since bryner is the developer who actually fixed the bug, our classifier-only prediction is inaccurate in this case. If we use the tossing graphs in addition to the classifier, we select the most likely tossee for bugzilla, the first developer in the classifier ranked list. In Figure 6, we present the node for bugzilla and its neighbors. ${ }^{8}$ If we rank the outgoing edges of bugzilla based on tossing probability alone, the bug should be tossed to developer ddahl. Though bryner has lower probability, he has committed patches to the product "Firefox" and component "General" that bug 254967 belong to. Therefore, our algorithm will choose bryner as the potential developer over $d d a h l$, and our prediction matches the actual bug fixer. Our ranking function also takes into account developer activity; in this example, however,

\footnotetext{
${ }^{7}$ Choosing 100 days as the threshold was based on Anvik et al. [9]'s observation that developers that have been inactive for three months or more are potentially retired.

${ }^{8}$ For clarity, we only present the nodes relevant to this example, and the labels at the point of validating this bug; due to incremental learning, label values will change over time.
} 
both developers ddahl and bryner are active, hence comparing their activities is not required. To conclude, our ranking function increases prediction accuracy while reducing tossing lengths; the actual tossing length for this particular Mozilla bug was 6 , and our technique reduces it to 2 .

We now describe our algorithm for ranking developers. Similar to Jeong et al., we first use the classifier to predict a set of developers named CP (Classifier Predicted). Using the last-activity information, we remove all developers who have not been active for the past 100 days from CP. We then sort the developers in CP using the fix counts from the developer profile (as described in Section 4.6.1).

Suppose the CP is $\left\{D_{1}, D_{2}, D_{3}, \ldots, D_{j}\right\}$. For each $D_{i}$ in the sorted CP, we rank its tossees $T_{k}$ (outgoing edges in the tossing graph) using the following ranking function:

\section{$\operatorname{Rank}\left(T_{k}\right)=\operatorname{Pr}\left(D_{i} \hookrightarrow T_{k}\right)+$ MatchedProduct $\left(T_{k}\right)+$ MatchedComponent $\left(T_{k}\right)+$ $\operatorname{LastActivity}\left(T_{k}\right)$}

The tossing probability, $\operatorname{Pr}\left(D_{i} \hookrightarrow T_{k}\right.$ ), is computed using equation 2 (Section 3). The function MatchedProduct $\left(T_{k}\right)$ returns 1 if the product the bug belongs to exists in developer $T_{k}$ 's profile, and 0 otherwise. Similarly, the function MatchedComponent $\left(T_{k}\right)$ returns 1 if the component the bug belongs to exists in developer $T_{k}$ 's profile. Note that the MatchedComponent $\left(T_{k}\right)$ attribute is computed only when MatchedProduct $\left(T_{k}\right)$ returns 1 . The LastActivity function returns 1 if $T_{k}$ 's last activity was in the last 100 days from the date the bug was reported. As a result, $0<\operatorname{Rank}\left(T_{k}\right) \leq 4$. We then sort the tossees $T_{k}$ by rank, choose the developer $T_{i}$ with highest rank and add it to the new set of potential developers, named ND. Thus after selecting $T_{i}$, where $i=1,2, \ldots, j$, the set ND becomes $\left\{D_{1}, T_{1}, D_{2}, T_{2}, D_{3}, T_{3}, \ldots, D_{j}, T_{j}\right\}$. When measuring our prediction accuracy, we use the first 5 developers in ND.

If two potential tossees $T_{i}$ and $T_{j}$ have the same rank, and both are active developers, and both have the same tossing probabilities for bug B (belonging to product $P$ and component $C$ ), we use developer profiles to further rank them. There can be two cases in this tie: (1) both $T_{i}$ and $T_{j}$ 's profiles contain $\{P, C\}$, or (2) there is no match with either $P$ or $C$. For the first case, consider the example in Table 3: suppose a new bug B belongs to $\left\{P_{1}, C_{1}\right\}$. Assume $T_{i}$ and $T_{j}$ are the two potential tossees from developer $D$ (where $D$ has been predicted by the classifier) and suppose both $T_{i}$ and $T_{j}$ have the same tossing probabilities from $D$. From developer profiles, we find that $T_{j}$ has fixed more bugs for $\left\{P_{1}, C_{1}\right\}$ than $T_{i}$, hence we choose $T_{j}$ (case 1 ). If the developers have the same fix count, or neither has $P$ and/or $C$ in their profile (case 2), we randomly choose one.

\subsection{Ablative Analysis for Tossing Graph Attributes}

As explained in Section 4.6.4, our ranking function for tossing graphs contains additional attributes compared to the original tossing graphs by Jeong et 


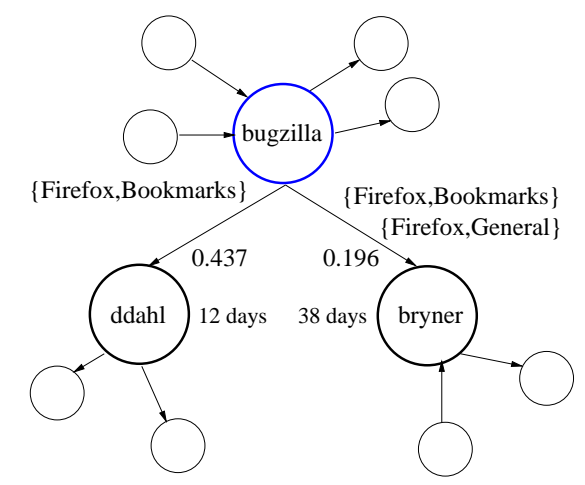

Figure 6: Actual multi-feature tossing graph extracted from Mozilla.

\begin{tabular}{|c||c|r|}
\hline Developer ID & Product-Component & Fix count \\
\hline$T_{i}$ & $\left\{P_{1}, C_{1}\right\}$ & 3 \\
& $\left\{P_{1}, C_{7}\right\}$ & 18 \\
& $\left\{P_{9}, C_{6}\right\}$ & 7 \\
\hline$T_{j}$ & $\left\{P_{1}, C_{1}\right\}$ & 13 \\
& $\left\{P_{4}, C_{6}\right\}$ & 11 \\
\hline
\end{tabular}

Table 3: Sample developer profiles: developer IDs and number of bugs they fixed in each product-component pair.

al. Therefore, we were interested to evaluate the importance of each attribute; to measure this, we performed another ablative analysis. We choose only two attributes out of three (product, component and developer activity) at a time and compute the decrease in prediction accuracy in the absence of the other attribute. For example, if we want to measure the significance of the "developer activity" attribute, we use only product and component attributes in our ranking function described in Section 4.6.4 and compute the decrease in prediction accuracy. In Section 5.5 we discuss the results of our ablative analysis and argue the importance of the attributes we propose.

\subsection{Accurate Yet Efficient Classification}

One of the primary disadvantages of fine-grained incremental learning is that it is time consuming $[26,27,28]$. Previous studies which used fine-grained incremental learning for other purposes [29] found that using a part of the bug repository history for classification might yield comparable and stable results to using the entire bug history. Similarly, we intended to find how many past bug reports we need to train the classifier on in order to achieve a prediction accuracy comparable to the highest prediction accuracy attained when using fold $1-10$ as the TDS and fold 11 as the VDS.

We now present the procedure we used for finding how much history is enough to yield high accuracy. We first built the tossing graphs using the TDS until fold 10; building tossing graphs and using them to rank developers is not 
a time consuming task, hence in our approach tossing graphs cover the entire TDS. We then incrementally started using sets of 5,000 bug reports from fold 10 downwards, in descending chronological order, as our TDS for the classifier, and measured our prediction accuracy for bugs in fold 11 (VDS); we continued this process until addition of bug reports did not improve the prediction accuracy any more, implying stabilization. Note that by this method our VDS remains constant. We present the results of our optimization in Section 5.7.

\subsection{Implementation}

In Figure 7 we compare our approach to previous techniques. Initial work in this area (Figure $7(\mathrm{a})$ ) used classifiers only $[9,10,11,12]$; more recent work by Jeong et al. [7] (Figure 7(b)) coupled classifiers with tossing graphs. Our approach (Figure 7(c)) adds fine-grained incremental learning and multi-feature tossing graphs. Our algorithm consists of four stages, as labeled in the figure: (1) initial classifier training and building the tossing graphs, (2) predicting potential developers, using the classifier and tossing graphs, (3) measuring prediction accuracy, (4) updating the training sets using the bugs which have been already validated, re-running the classifier and updating the tossing graphs. We iterate these four steps until all bugs have been validated.

\subsubsection{Developer Profiles}

\begin{tabular}{|c||c|r|}
\hline Developer ID & Product-Component & Fix count \\
\hline$D_{1}$ & $\left\{P_{1}, C_{2}\right\}$ & 3 \\
& $\left\{P_{1}, C_{7}\right\}$ & 18 \\
& $\left\{P_{9}, C_{6}\right\}$ & 7 \\
\hline
\end{tabular}

Table 4: Sample developer profile.

We maintain a list of all developers and their history of bug fixes. Each developer $D$ has a list of product-component pairs $\{P, C\}$ and their absolute count attached to his or her profile. A sample developer profile is shown in Table 4, e.g., developer $D_{1}$ has fixed 3 bugs associated with product $P_{1}$ and component $C_{2}$. This information is useful beyond bug assignments; for example, while choosing moderators for a specific product or component it is a common practice to refer to the developer performance and familiarity with that product or component.

\subsubsection{Classification}

Given a new bug report, the classifier produces a set of potential developers who could fix the bug. We describe the classification process in the remainder of this subsection.

Choosing fixed bug reports. We use the same heuristics as Anvik et al. [9] for obtaining fixed bug reports from all bug reports in Bugzilla. First, we extract all bugs marked as "verified" or "resolved"; next, we remove all bugs marked 


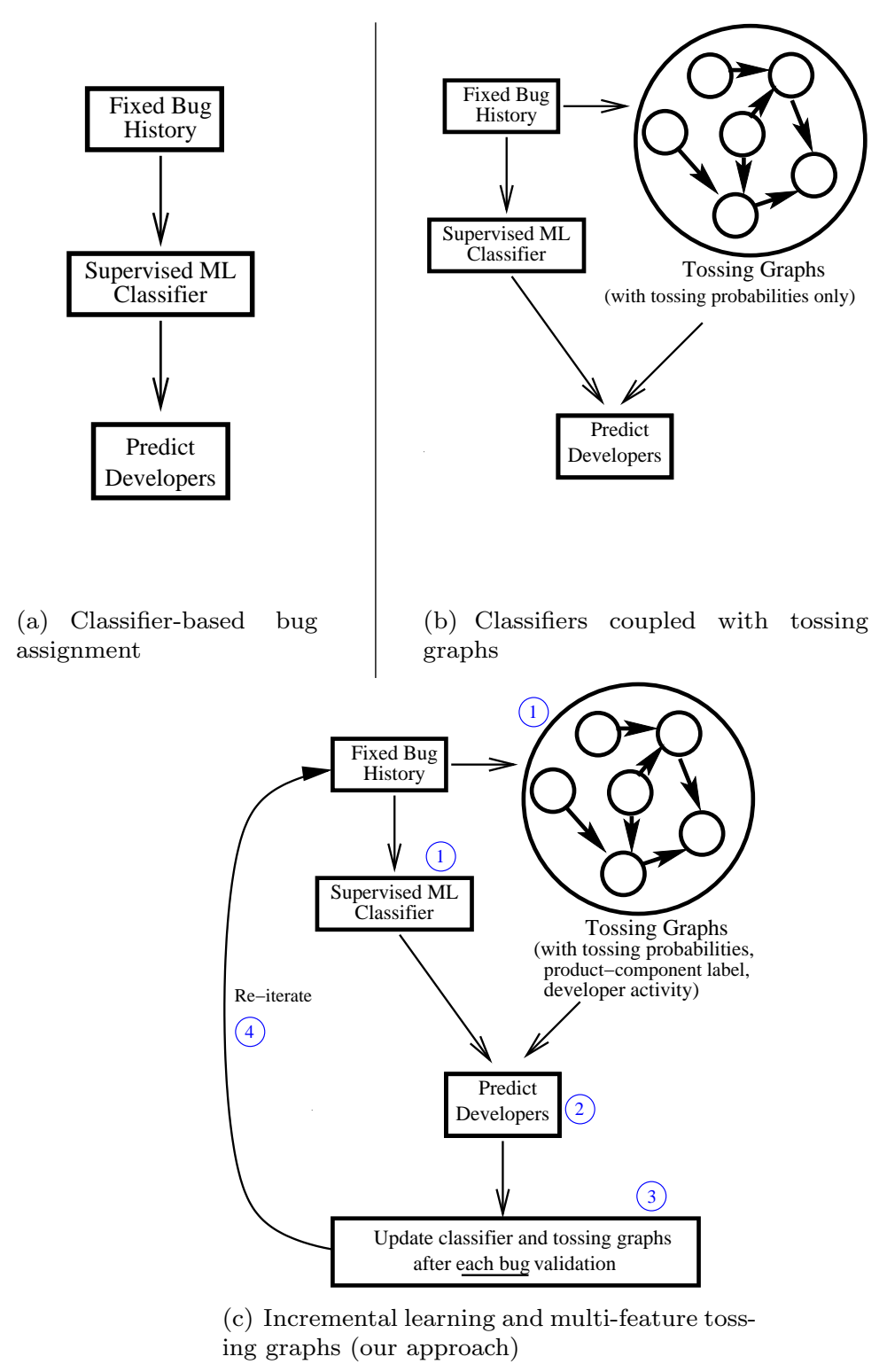

Figure 7: Comparison of bug assignment techniques.

as "duplicate" or "works-for-me," which leaves us with the set containing fixed bugs only.

Accumulating training data. Prior work $[9,10,11]$ has used keywords from the bug report and developer name or ID as attributes for the training data sets; we also include the product and component the bug belongs to. For extracting 
relevant words from bug reports, we employ tf-idf, stemming, stop-word and non-alphabetic word removal [19].

Filtering developers for classifier training. Anvik et al. refine the set of training reports by using several heuristics. For example, they do not consider developers who fixed a small number of bugs, which helps remove noise from the TDS. Although this is an effective way to filter non-experts from the training data and improve accuracy, in our approach filtering is unnecessary: the ranking function is designed such that, if there are two developers $A$ and $B$ who have fixed bugs of the same class $K$, but the number of $K$-type bugs $A$ has fixed is greater than the number of $K$-type bugs $B$ has fixed, a $K$-type bug will be assigned to $A$.

\subsubsection{Multi-feature Tossing Graphs}

With the training data and classifier at hand, we proceed to constructing tossing graphs as explained in Section 4.3.1. We use the same bug reports used for classification to build the tossing graphs.

Filtering developers for building tossing graphs. We do not prune the tossing graphs based on a pre-defined minimum support (frequency of contribution) for a developer, or the minimum number of tosses between two developers. Jeong et al. [7] discuss the significance of removing developers who fixed less than 10 bugs and pruning edges between developers that have less than $15 \%$ transaction probability. Since their approach uses the probability of tossing alone to rank neighboring developers, they need the minimum support values to prune the graph. In contrast, the multiple features in our tossing graphs coupled with the ranking function (as explained in the Section 4.6.4) obviate the need for pruning.

\subsubsection{Predicting Developers}

For each bug, we predict potential developers using two methods: (1) using the classifier alone, to demonstrate the advantages of incremental learning, and (2) using both the classifier and tossing graphs, to show the significance of multifeature tossing graphs. When using the classifier alone, the input consists of bug keywords, and the classifier returns a list of developers ranked by relevance; we select the top five from this list. When using the classifier in conjunction with tossing graphs, we select the top three developers from this list, then for developers ranked 1 and 2 we use the tossing graph to recommend a potential tossee, similar to Jeong et al. For predicting potential tossees based on the tossing graph, our tossee ranking function takes into account multiple factors, in addition to the tossing probability as proposed by Jeong et al. In particular, our ranking function is also dependent on (1) the product and component of the bug, and (2) the last activity of a developer, to filter retired developers. Thus our final list of predicted developers contains five developer id's in both methods (classifier alone and classifier + tossing graph). 


\subsubsection{Folding}

After predicting developers, similar to the Bettenburg et al.'s folding technique [11], we iterate the training and validation for all folds. However, since our classifier and tossing graph updates are already performed during validation, we do not have to update our training data sets after each fold validation. To maintain consistency in comparing our prediction accuracies with previous approaches, we report the average prediction accuracy over each fold.

\section{Results}

\subsection{Experimental Setup}

We used Mozilla and Eclipse bugs to measure the accuracy of our proposed algorithm. We analyzed the entire life span of both applications. For Mozilla, our data set ranges from bug number 37 to 549,999 (May 1998 to March 2010). For Eclipse, we considered bugs numbers from 1 to 306,296 (October 2001 to March 2010). Mozilla and Eclipse bug reports have been found to be of high quality [7], which helps reduce noise when training the classifiers. We divided our bug data sets into 11 folds and executed 10 iterations to cover all the folds.

Data collection. We used the bug reports to collect four kinds of data:

1. Keywords: we collect keywords from the bug title, bug description and comments in the bug report.

2. Bug source: we retrieve the product and component the bug has been filed under from the bug report.

3. Temporal information: we collect information about when the bug has been reported and when it has been been fixed.

4. Developers assigned: we collect the list of developer IDs assigned to the bug from the activity page of the bug and the bug routing sequence.

\subsection{Prediction Accuracy}

In Tables 5 and 6 we show the results for predicting potential developers who can fix a bug for Mozilla and Eclipse using five classifiers: Naïve Bayes, Bayesian Networks, C4.5, and SVM using Polynomial and RBF kernel functions. In our experiments, we used the classifier implementations in Weka for the first three classifiers [25] and WLSVM for SVM [30]. ${ }^{9}$

\footnotetext{
${ }^{9}$ The details of the parameters used for the classifiers in the experiments can be found at: http://www.cs.ucr.edu/ neamtiu/bugassignment-params/
} 


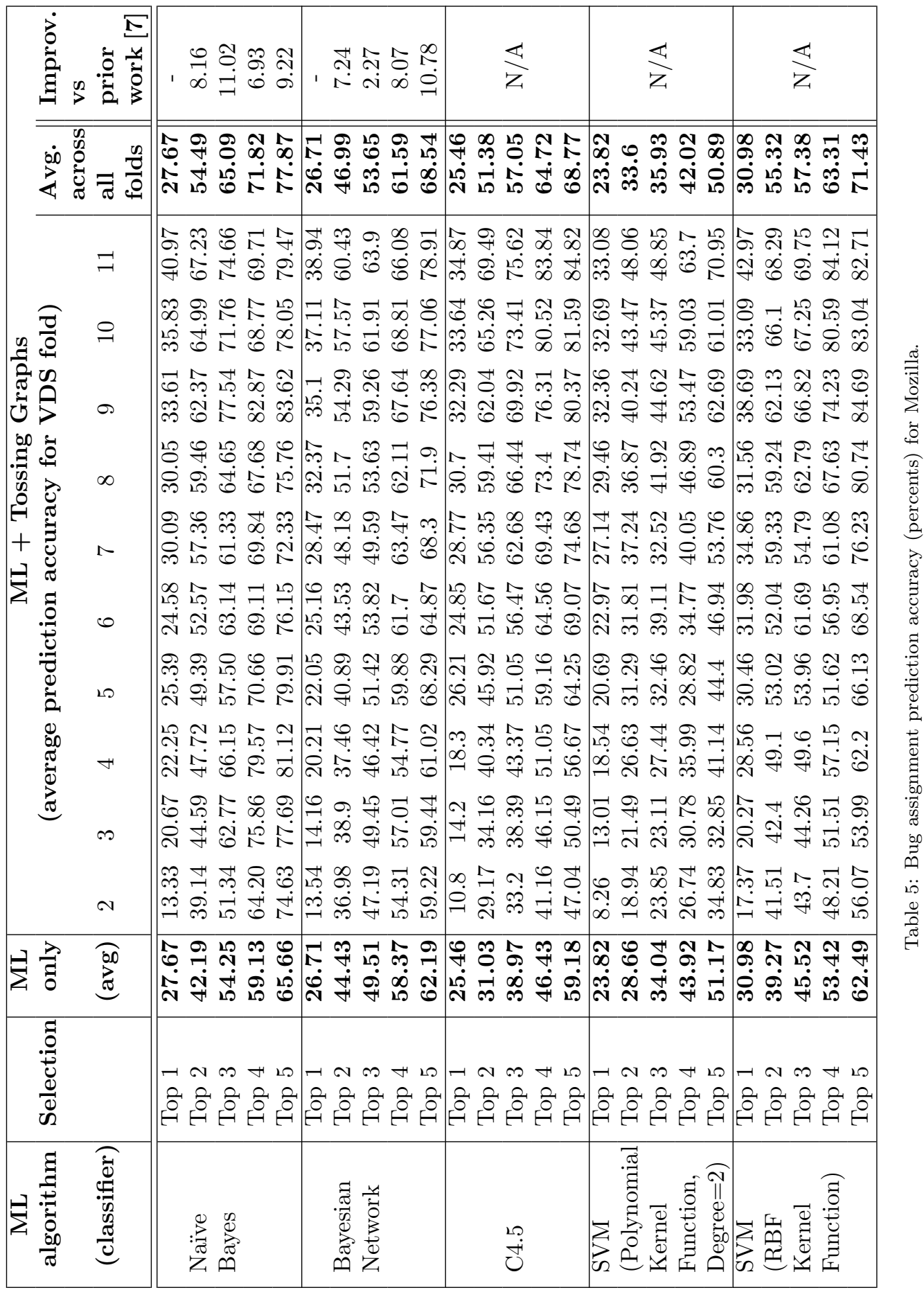




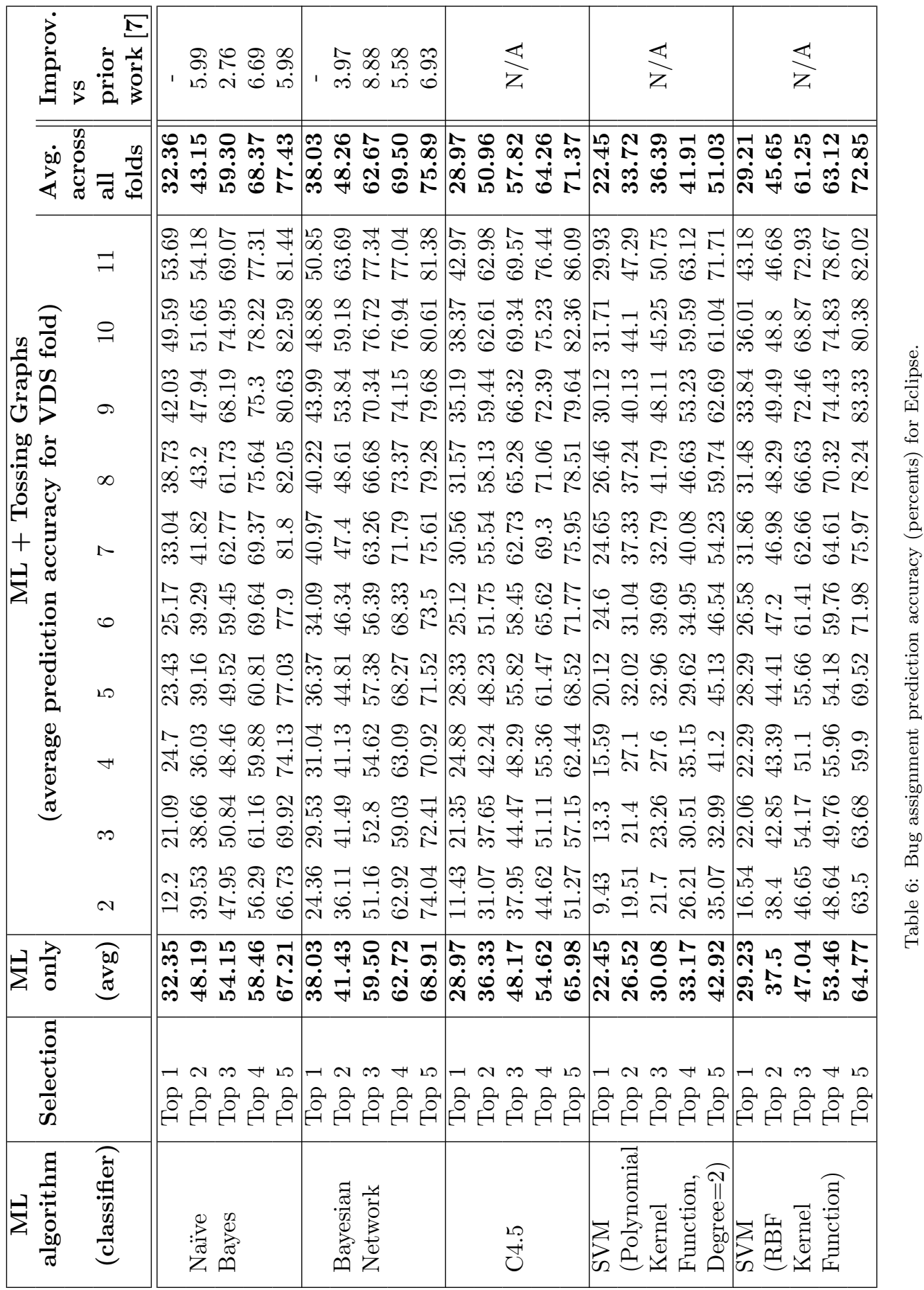


Classifier alone. To demonstrate the advantage of our fine-grained, incremental learning approach, we measure the prediction accuracy of the classifier alone; column "ML only" contains the classifier-only average prediction accuracy rate. We found that, for Eclipse and Mozilla, our approach increases accuracy by 8.91 percentage points on average compared to the best previously-reported, no-incremental learning approach, by Anvik et al. [9]. This confirms that incremental learning is instrumental for achieving a high prediction accuracy. Anvik et al. report that their initial investigation of incremental learning did not yield highly accurate predictions, though no details are provided. Note that we use different data sets (their experiments are based on 8,655 reports for Eclipse and 9,752 for Firefox, while we use 306,297 reports for Eclipse and 549,962 reports for Mozilla) and additional attributes for training and validation.

Classifier + tossing graphs. Columns "ML+Tossing Graphs" of Tables 5 and 6 contain the average accurate predictions for each fold (Top 2 to Top 5 developers) when using both the classifier and the tossing graph; the Top 1 developer is predicted using the classifier only. Consider row 2, which contains prediction accuracy results for Top 2 in Mozilla using the Naïve Bayes classifier: column 4 (value 39.14) represents the percentage of correct predictions for fold 1 ; column 5 (value 44.59) represents the percentage of correct predictions for folds 1 and 2; column 14 (value 54.49) represents the average value for all iterations across all folds. Column 15 represents the percentage improvement of prediction accuracy obtained by our technique when compared to using tossing graphs with tossing probabilities only. Our best average accuracy is achieved using Naïve Bayes ( $77.87 \%$ for Mozilla and $77.43 \%$ for Eclipse). We found that this prediction accuracy is higher than the prediction accuracy we obtained in our earlier work [31] where we used Naïve Bayes and Bayesian Networks only. When compared to prior work [7] (where Naïve Bayes and Bayesian Networks were used as ML algorithms and tossing probabilities alone were used in the tossing graphs) our technique improved prediction accuracy by up to 11.02 percentage points. However, when measuring across the average of all ten folds, our model achieved highest prediction accuracy of $77.87 \%$ for Mozilla using Naïve Bayes and $75.89 \%$ for Eclipse using Bayesian Networks. The last column shows the percentage increase in prediction accuracy from using single-attribute tossing graphs with tossing probability alone [7] compared to our approach in which we used a ranking function based on the multi-attribute tossing graphs we proposed.

Classifier selection. In Section 4.1.1 we discussed that one of the objectives of using a broad range of classifiers for evaluating our framework is to analyze if a particular classifier is best suited for the bug assignment problem. Our results in Tables 5 and 6 reveal that the answer is complex. Generally, Naïve Bayes works best for early VDS folds (when there are fewer data) and when considering Top 4 or Top 5 accuracies. The polynomial-kernel SVM performs fairly poorly. The other three are comparable, without an obvious pattern.

Our results are consistent with the standard statistical learning theory of bias-variance [32]. In particular, with fewer data (or more noise in the data) 
better results are achieved by using a less flexible classifier (one with fewer parameters and more bias). This supports the performance of Naïve Bayes: it does better for small sample sizes and in case where the testing metric does not match the training metric as well (Top 5, for instance) which looks like noisier data. Additionally, if the bias is too far from the true answer, the method will not work well. The polynomial-kernel SVM probably has such a mismatch: its bias is too far from the correct bug triage classifier. In particular, it is a global classifier in that all training data affect the classifications for all inputs. By contrast, C4.5 and RBF SVM both are local classifiers: only training data near the testing point have a large influence on the resulting classification. This suggests that local classification methods will do best on bug assignment.

Among the more flexible (less biased) local classifiers (Bayesian networks, C4.5, and RBF SVM), there is not a clear winner - all seem equally well suited for bug assignment. On any particular task, one will do better than the others, but a systematic prediction about other tasks cannot be made from these experiments: much will depend on the amount of data, and the noise present. All of these methods have "regularization parameters" that can adjust the amount of bias. Picking a suitable value based on the amount of data and noise is more important for achieving good results than the exact classifier used.

\subsection{Tossing Length Reduction}

We compute the original tossing path lengths for "fixed" bugs in Mozilla and Eclipse, and present them in Figure 8; we observe that most bugs have tossing length less than 13 for both applications. Note that tossing length is zero if the first assigned developer is able to resolve the bug. Ideally, a bug assignment model should be able to recommend bug fixers such that tossing lengths are zero. However, this is unlikely to happen in practice due to the unique nature of bugs. Though Jeong et al. measured tossing lengths for both "assigned" and "verified" bugs, we ignore "assigned" bugs because they are still open, hence we do not have ground truth (we do not know the final tossing length yet). In Figure 9, we present the average reduced tossing lengths of the bugs for which we could correctly predict the developer. We find that the predicted tossing lengths are reduced significantly, especially for bugs which have original tossing lengths less than 13. Our approach reports reductions in tossing lengths by up to $86.67 \%$ in Mozilla and $83.28 \%$ in Eclipse. For correctly-predicted bugs with original tossing length less than 13, prior work [7] has reduced tossing path lengths to 2-4 tosses, while our approach reduces them to an average of 1.5 tosses for Mozilla and 1.8 tosses for Eclipse, hence multi-feature tossing graphs prove to be very effective.

\subsection{Filtering Noise in Bug Reports}

We found that when training sets comprise bugs with resolution "verified" or "resolved" and arbitrary status, the noise is much higher than when considering bugs with resolution "verified" or "resolved" and status "fixed". In fact, we found that, when considering arbitrary-status bugs, the accuracy is on average 


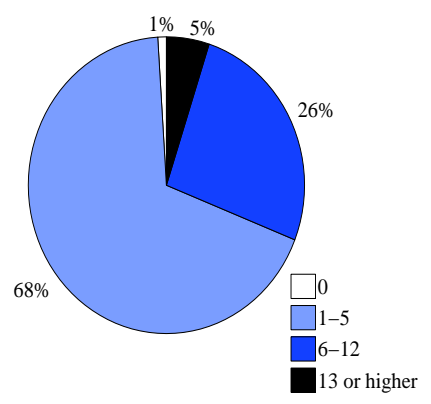

(a) Mozilla

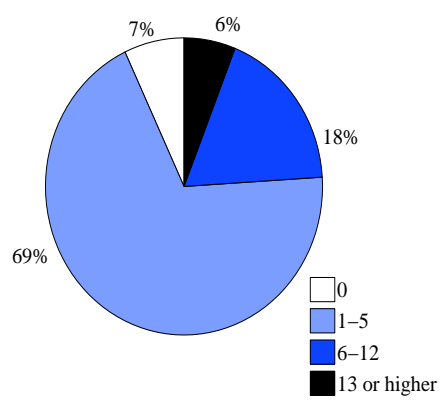

(b) Eclipse

Figure 8: Original tossing length distribution for "fixed" bugs.

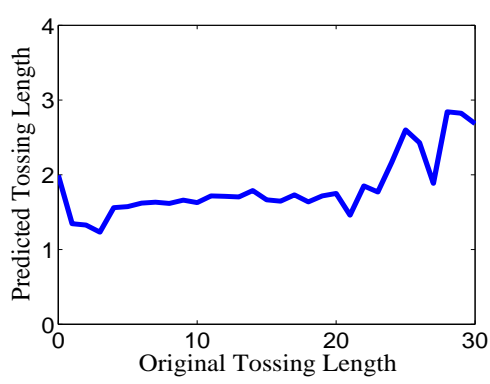

(a) Mozilla

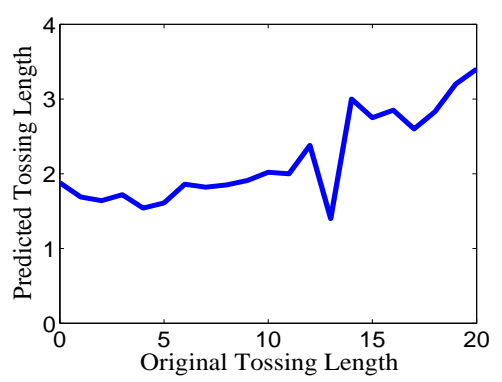

(b) Eclipse

Figure 9: Average reduction in tossing lengths for correctly predicted bugs when using ML + Tossing Graphs (using both classifiers).

$23 \%$ lower than the accuracy attained when considering fixed-status bugs only. Jeong et al. considered all bugs with resolution "verified" and arbitrary-status for their training and validation purposes. They found that tossing graphs are noisy, hence they chose to prune developers with support less than 10 and edges with transaction probability less than $15 \%$.

Our analysis suggests that bugs whose status changes from "new" or "open" to "fixed" are actual bugs which have been resolved, even though various other kinds of bugs, such as "invalid," "works-for-me," "wontfix," "incomplete" or "duplicate" may be categorized as "verified" or "resolved." We conjecture that developers who submit patches are more competent than developers who only verify the validity of a bug and mark them as "invalid" or developers who find a temporary solution and change the bug status to "works-for-me." Anvik et al. made a similar distinction between message repliers and contributors/maintainers; they found that only a subset of those replying to bug messages are actually submitting patches and contributing to the source code, hence they only retain the contributing repliers for their TDS. 


\begin{tabular}{|c|c|c|c|c|}
\hline Project & Selection & \multicolumn{3}{|c|}{ Average Prediction Accuracy (\%) } \\
\cline { 3 - 5 } & & $\begin{array}{l}\text { With intra- } \\
\text { and inter-fold } \\
\text { updates (best) }\end{array}$ & $\begin{array}{l}\text { Without } \\
\text { intra-fold } \\
\text { updates }\end{array}$ & $\begin{array}{l}\text { Without } \\
\text { inter-fold } \\
\text { updates }\end{array}$ \\
\hline \hline \multirow{5}{*}{ Mozilla } & Top 1 & 27.67 & $13.53(-14.15)$ & $7.86(-19.82)$ \\
& Top 2 & 54.49 & $26.59(-27.90)$ & $12.54(-41.95)$ \\
& Top 3 & 65.09 & $47.20(-17.88)$ & $28.61(-36.48)$ \\
& Top 4 & 71.82 & $53.24(-18.60)$ & $36.63(-35.2)$ \\
& Top 5 & 77.87 & $62.22(-15.66)$ & $43.86(-34.02)$ \\
\hline \hline \multirow{5}{*}{ Eclipse } & Top 1 & 32.36 & $8.64(-23.73)$ & $11.43(-20.94)$ \\
& Top 2 & 43.15 & $19.18(-23.97)$ & $16.02(-27.13)$ \\
& Top 3 & 59.30 & $32.82(-26.48)$ & $27.15(-32.15)$ \\
& Top 4 & 68.37 & $44.30(-24.07)$ & $32.49(-35.88)$ \\
& Top 5 & 77.43 & $58.33(-19.10)$ & $39.47(-37.96)$ \\
\hline
\end{tabular}

Table 7: Impact of inter- and intra-folding on prediction accuracy using Naïve Bayes classifier.

\subsection{Importance of Individual Tossing Graph Attributes}

Since our ranking function for tossing graphs contains additional attributes compared to the original tossing graphs by Jeong et al., we were interested in evaluating the importance of each attribute using ablative analysis as described in Section 4.4. Therefore, we compute, for each fold, the reduction in accuracy caused by removing one attribute from the ranking function and keeping the other two. In Figure 10 we show the minimum (bottom black bar), maximum (top black bar) and average (red bar) across all folds. The decrease in prediction accuracy shows that the removal of product and developer activity attributes affects the prediction accuracy the most. These accuracy reductions underline the importance of using all attributes in the ranking function, and more generally, the advantage of the richer feature vectors our approach relies on. Note that removing developer activity affects prediction accuracy in Mozilla more significantly than in Eclipse. Analyzing the significance of each attribute in our ranking function for individual projects, i.e., build a ranking function per project, is beyond the scope of this paper.

\subsection{Importance of Incremental Learning}

To assess the significance of incremental learning in our technique, we performed two sets of experiments. We took our best results, obtained as shown in Figure 1, i.e., using Naïve Bayes classifier, with tossing graphs, productcomponent and incremental learning, and then unilaterally varied the learning procedure. ${ }^{10}$ The best-result data has been shown in Tables 5 and 6 but for ease of comparison we report the same data in shown column 3 of Table 7 .

\footnotetext{
${ }^{10}$ We chose Naïve Bayes since the average prediction accuracy was highest for this classifier compared to other classifiers, hence, consistent with the standard machine learning practice of ablative analysis, we varied incremental learning to quantify its impact.
} 
Intra-fold updates. To evaluate the impact of disabling of intra-fold updates we also trained our model using folds 1 to $(N-1)$ and we used fold $N$ for prediction. The results of the average prediction accuracy are presented in column 4 of Table 7 . For example, our results show that for Top 1 developers in Mozilla, the average prediction accuracy across 10 folds is $13.53 \%$, a decrease of 14.15 percentage points when compared to the incremental learning (inter- and intra-fold updates) technique shown in column 3.

Inter-fold updates. To evaluate the importance of inter-fold updates for each fold, we first trained our model using the first $80 \%$ of the bug reports in that fold only. Next, we used the remaining $20 \%$ of the bug reports in that fold only for measuring prediction accuracy. Note that in this case, the bug reports from folds 1 to $N-1$ are not added to fold $N$ while training the classifier. The average prediction accuracy is presented in column 5 of Table 7. For example, our results show that for Top 1 developers in Mozilla, the average prediction accuracy across 10 folds is $7.86 \%$, a decrease of $19.82 \%$ when compared to the incremental learning (inter- and intra-fold updates) technique shown in column 3.

Conclusions. The results in Table 7 suggest there is a significant decrease in prediction accuracy (up to $42 \%$ ) when incremental learning (inter- and intra-fold updates) is removed from our algorithm. This reduction in prediction accuracy suggests that indeed incremental learning is instrumental to achieving higher prediction accuracy for bug assignment: inter-and intra-folding lead to tossing graphs with highly accurate transaction probabilities which, helps improve our prediction accuracy. Note that incremental learning (or folding) is not a contribution of our work; incremental learning is a standard technique to improve the prediction accuracy in any supervised or unsupervised learning algorithms in machine learning [24]. Rather, these experiments were performed to demonstrate that in comparison to prior work, where split-sample validation was used, automatic bug assignment can benefit significantly from incremental learning.

\subsection{Accurate Yet Efficient Classification}

One of the primary disadvantages of fine-grained incremental learning is that it is very time consuming. As described in Section 4.5, we performed a study to find how many past bug reports we need to train the classifier to achieve approximately similar prediction accuracy when compared to the highest prediction accuracy attained when using folds 1-10 as the TDS and fold 11 as the VDS. We used the Naïve Bayes classifier as our ML algorithm in this case. We present our results in Figure 11. We found that Mozilla required approximately $14 \%$ and Eclipse required about $26 \%$ of all bug reports (in reverse chronological order, i.e., most recent bugs) to achieve prediction accuracies greater than $80 \%-$ within 5 percentage points of the best results of our original experiments where we used the complete bug history to train our classifier. Therefore, a practical way to reduce the computational effort associated with learning, yet maintain high prediction accuracy, is to prune the bug report set and only use a recent 


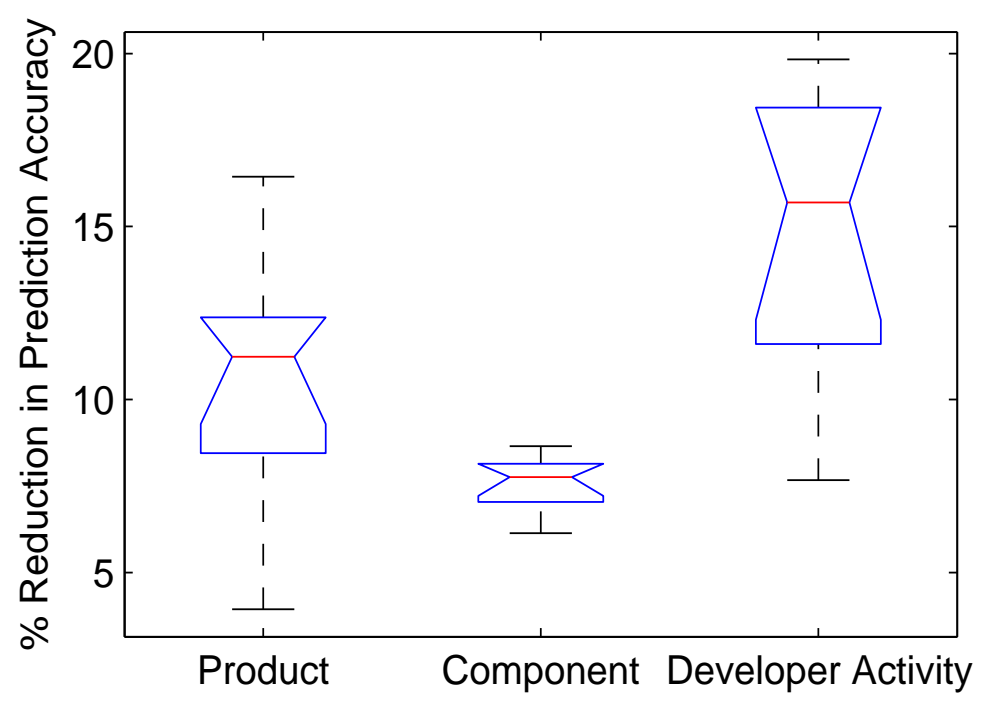

(a) Mozilla

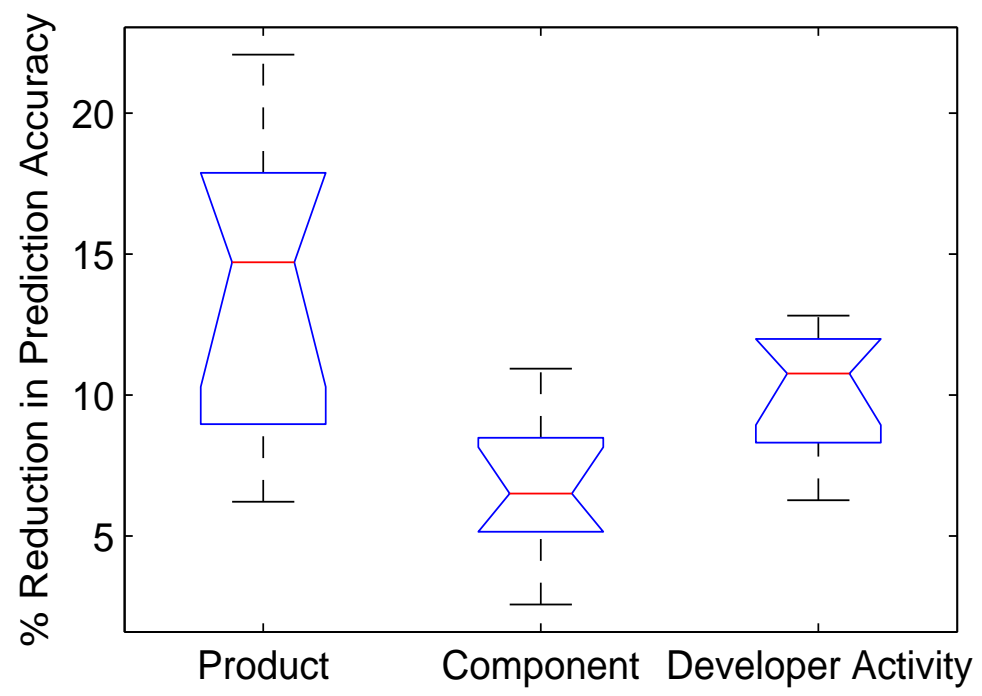

(b) Eclipse

Figure 10: Impact of individual ranking function attributes on prediction accuracy.

subset (e.g., the most recent $14 \%$ to $26 \%$ of bug reports, depending on the project). 


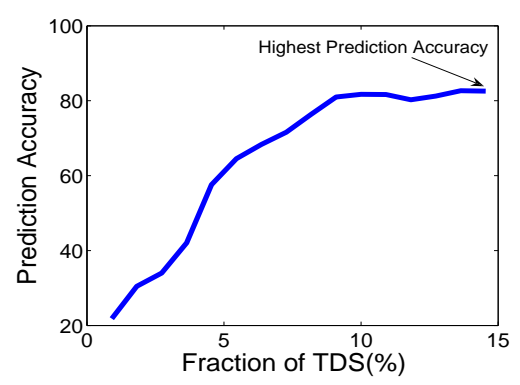

(a) Mozilla

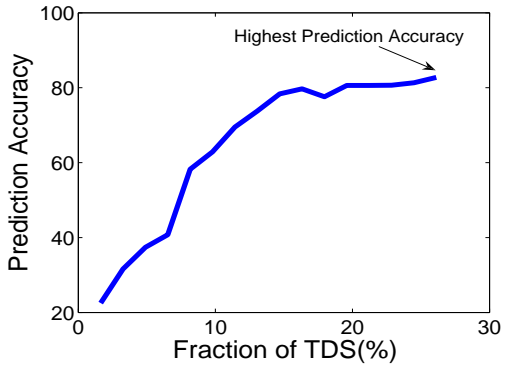

(b) Eclipse

Figure 11: Change in prediction accuracy when using subsets of bug reports using Naïve Bayes classifier.

Computational effort. The intra-fold updates used in our approach are more computationally-intensive than inter-fold updates. However, for practical purposes this is not a concern because very few bugs get fixed the day they are reported. Before we use the algorithm to predict developers, we train it with all fixed bug reports in the history; when a new bug gets fixed, the TDS needs to be updated and we need to re-train the classifier. However, while about 100 bugs are reported every day for large projects like Mozilla and Eclipse, less than 1 bug gets fixed every day, on average [7]. Since we use fixed bug reports only, if we update the TDS overnight with the new fixed bug reports and retrain the classifier, we can still achieve high prediction accuracies.

\section{Threats To Validity}

We now present possible threats to the validity of our study.

\subsection{Internal Validity}

In our study we collected bug reports from Bugzilla for both Eclipse and Mozilla. Bug reports can have various status at a given point in time: "unconfirmed," "new," "assigned," "reopened," "resolved," "verified," and "closed". A bug which has status resolution status as "fixed" can be either "verified" or "closed" at a given point. For our training and validation purposes, we look at bugs which have the resolution status as fixed irrespective of whether it is "verified" or "closed". We filter our data set to fixed bugs only for the following reasons: (1) for bugs which are unconfirmed, it is not possible to say if they are indeed bugs, (2) for new bugs it is not known who the developer will be who will fix that bug and hence these bugs cannot be used for training a supervised classifier where the end-result knowledge is necessary, (3) reopened bugs are similar to new bugs and hence are not a part of our training/validation, (4) resolved bugs are those for which a resolution has been provided by a developer but is still in the review process which implies that the bug might be re-assigned (or tossed) if the resolution is not satisfactory. For accurate supervised learning, we 
need to ensure that the training set includes the correct expertise of the developers. One potential threat to validity in our study is that a bug $B$ which has been fixed and closed can be reopened at a later time. In that case developer $D$ who earlier resolved bug $B$ might not resolve the issues with reopening the bug again and might affect our classification results. However, it is impossible to predict what percentage of currently-fixed bugs will be reopened in future and quantify the effects of bug reopening on our results. Another potential threats to validity in our study is not differentiating between bugs and enhancement requests.

\subsection{External Validity}

Generalization to other systems. The high quality of bug reports found in Mozilla and Eclipse [7] facilitates the use of classification methods. However, we cannot claim that our findings generalize to bug databases for other projects. Additionally, we have validated our approach on open source projects only, but commercial software might have different assignment policies and we might require considering different attribute sets.

Small projects. We used two large and widely-used open source projects for our experiments, Mozilla and Eclipse. Both projects have multiple products and components, hence we could use this information as attributes for our classifier and labels in our tossing graphs. For comparatively smaller projects which do not have products or components, the lack of product-component labels on edges would reduce accuracy. Additionally, for smaller projects the 90-days heuristic we use for pruning inactive developers might have to change. In the future when we analyze smaller projects, we plan to empirically study the average lifetime of a developer for the project to determine inactive and active developers. Never-

theless, fine-grained incremental learning and pruning inactive developers would still be beneficial.

\subsection{Construct Validity}

For the projects we used, we did not differentiate between various roles (e.g., developers, triagers, managers) contributors serve in the project. Our approach neither divides contributors according to the roles they play in the community, nor ranks them higher based on their familiarity with the source code. In the future, we plan to include developer's source code expertise in the future to further improve our ranking function. Additionally, it is not possible to find out in our framework if the first developer who was assigned the bug was a default assignee or assigned by the triager explicitly for any projects. However, for the projects we chose - Mozilla and Eclipse - developers were cc'ed by default when they are responsible for a specific product or component, but they are not assigned the bug by default for fixing it. 


\subsection{Content Validity}

Information retrieval and learning tools. We used Weka for extracting relevant keywords after stop-word removal and tf-idf as explained in Section 4.6. We also used the built-in classifiers of Weka and LibSVM for learning our model. Hence, another potential threat to validity is error in these tools or how changes in implementation of these classifiers might affect our results.

Developer identity. The assignee information in Bugzilla does not contain the domain info of the email address for a developer. Therefore, we could not differentiate between users with same email id but different domains. For instance, in our technique, bugzilla@alice.com, and bugzilla@bob.com will be in the same bucket as bugzilla@standard8.plus.com. This might potentially lead to inaccurate predictions and decrease the prediction accuracy of our model.

Load balancing. Our technique does not consider load balancing while assigning bugs to developers. This is a potential threat to validity in the following sense: if our approach predicts that developer $D$ is the best match to fix a bug, he/she might be overloaded, so assigning them another bug might increase the bug-fix time.

\section{Conclusions}

Machine learning and tossing graphs have proved to be promising for automating bug assignment. In this paper we lay the foundation for future work that uses machine learning techniques to improve automatic bug assignment by examining the impact of multiple machine learning dimensions-learning strategy, attributes, classifiers - on assignment accuracy.

We used a broad range of text classifiers and found that, unlike many problems which use specific machine learning algorithms, we could not select a specific classifier for the bug assignment problem. We show that, for bug assignment, computationally-intensive classification algorithms such as C4.5 and SVM do not always perform better than their simple counterparts such as Naïve Bayes and Bayesian Networks. We performed an ablative analysis to measure the relative importance of various software process attributes in prediction accuracy. Our study indicates that to avoid the time-consuming classification process we can use a subset of the bug reports from the bug databases and yet achieve stable-high prediction accuracy.

We validated our approach on two large, long-lived open-source projects; in the future, we plan to test how our current model generalizes to projects of different scale and lifespan. In particular we would like to find if the classifier preference should change as the project evolves and how source code familiarity of a developer could be used as an additional attribute for ranking developers. Similarly, when we assign tossing probabilities, we only consider the developer who could finally fix the bug. However, it is common that developers contribute partially to the final patch in various ways. For example, when a bug is assigned to a developer, he might provide insights and add notes to the bug report 
instead of actually fixing the bug; in fact, there are contributors who provide useful discussions about a bug in the comment sections of a bug report who are never associated with the fixing process directly. These contributions are not considered in our ranking process, though they would significantly help in understanding contributor expertise and role in the software development community. Quantifying how these useful insights (or contribution) can be attributed towards the bug-fix based expertise of a contributor has the potential of further improving the triaging process. We also intend to test our approach on proprietary software where developer expertise, role, and contributions are more clearly defined.

\section{Acknowledgements}

This research was supported in part by NSF grant CCF-1149632. We thank the anonymous referees for their helpful comments on this paper.

\section{References}

[1] NIST, The economic impacts of inadequate infrastructure for software testing, Planning Report, 2002.

[2] J. Koskinen, http://users.jyu.fi/〜koskinen/smcosts.htm, 2003.

[3] R. C. Seacord, D. Plakosh, G. A. Lewis, Modernizing Legacy Systems: Software Technologies, Engineering Process and Business Practices, AddisonWesley, ISBN 0321118847, 2003.

[4] I. Sommerville, Software Engineering (7th Edition), Pearson Addison Wesley, ISBN 0321210263, 2004.

[5] Bugzilla User Database, http://www.bugzilla.org/ installation-list/, 2010.

[6] Increase in Open Source Growth, http:// sof tware.intel.com/en-us/blogs/2009/08/04/ idc-reports-an-increase-in-open-source-growth/, 2009.

[7] G. Jeong, S. Kim, T. Zimmermann, Improving Bug Triage with Bug Tossing Graphs, in: FSE, 2009.

[8] I. Witten, E. Frank, Data Mining: Practical Machine Learning Tools and Techniques, Morgan Kaufmann, second edn., 2005.

[9] J. Anvik, L. Hiew, G. C. Murphy, Who should fix this bug?, in: ICSE, 361-370, 2006.

[10] D. Cubranic, G. C. Murphy, Automatic bug triage using text categorization, in: SEKE, 2004. 
[11] N. Bettenburg, R. Premraj, T. Zimmermann, S. Kim, Duplicate Bug Reports Considered Harmful... Really?, in: ICSM, 2008.

[12] G. Canfora, L. Cerulo, Supporting change request assignment in open source development, in: SAC, 1767-1772, 2006.

[13] J. K. Anvik, Assisting Bug Report Triage through Recommendation, Ph.D. thesis, University of British Columbia, 2007.

[14] G. Canfora, L. Cerulo, How software repositories can help in resolving a new change request, in: Workshop on Empirical Studies in Reverse Engineering, 2005 .

[15] A. Podgurski, D. Leon, P. Francis, W. Masri, M. Minch, J. Sun, B. Wang, Automated support for classifying software failure reports, in: ICSE, 465$475,2003$.

[16] Z. Lin, F. Shu, Y. Yang, C. Hu, Q. Wang, An empirical study on bug assignment automation using Chinese bug data, in: ESEM, 2009.

[17] G. A. D. Lucca, M. D. Penta, S. Gradara, An Approach to Classify Software Maintenance Requests, in: ICSM, 93-102, 2002.

[18] D. Matter, A. Kuhn, O. Nierstrasz, Assigning bug reports using a vocabulary-based expertise model of developers, MSR .

[19] C. D. Manning, P. Raghavan, H. Schtze, Introduction to Information Retrieval, Cambridge University Press, 2008.

[20] P. Domingos, M. Pazzani, Beyond Independence: Conditions for the Optimality of the Simple Bayesian Classifier, in: Machine Learning, Morgan Kaufmann, 105-112, 1996.

[21] D. Koller, N. Friedman, Probabilistic Graphical Models: Principles and Techniques, The MIT Press, 2009.

[22] J. R. Quinlan, C4.5: programs for machine learning, Morgan Kaufmann Publishers Inc., San Francisco, CA, USA, ISBN 1-55860-238-0, 1993.

[23] B. E. Boser, I. M. Guyon, V. N. Vapnik, A Training Algorithm for Optimal Margin Classifiers, in: Proceedings of the Fifth Annual Workshop on Computational Learning Theory, 144-152, 1992.

[24] R. Kohavi, A Study of Cross-Validation and Bootstrap for Accuracy Estimation and Model Selection, Morgan Kaufmann, 1995.

[25] Weka Toolkit 3.6, http://www.cs.waikato.ac.nz/ml/weka/, 2010.

[26] E. Osuna, R. Freund, F. Girosi, An improved training algorithm for support vector machines, in: IEEE Workshop on Neural Networks for Signal Processing, 1997. 
[27] J. C. Platt, Fast training of support vector machines using sequential minimal optimization, in: In Advances in kernel methods: support vector learning, 1999.

[28] C. time in cross validation, http://en.wikipedia.org/wiki/ Cross-validationstatisticsComputational_issues, 2010.

[29] A. Lamkanfi, S. Demeyer, E. Giger, B. Goethals, Predicting the severity of a reported bug, in: MSR, 1-10, 2010.

[30] Y. EL-Manzalawy, V. Honavar, WLSVM: Integrating LibSVM into Weka Environment, Software available at http://www.cs.iastate.edu/ yasser/wlsvm, 2005.

[31] P. Bhattacharya, I. Neamtiu, Fine-grained Incremental Learning and Multifeature Tossing Graphs to Improve Bug Triaging, in: IEEE Conference on Software Maintenance, 2010.

[32] T. Hastie, R. Tibshirani, J. Friedman, The Elements of Statistical Learning: Data Mining, Inference, and Prediction, Springer, second edn., 2009. 